\title{
EXISTENCE AND UNIQUENESS OF SOLUTIONS TO DYNAMICAL UNILATERAL CONTACT PROBLEMS WITH COULOMB FRICTION: THE CASE OF A COLLECTION OF POINTS
}

\author{
${\text { Alexandre } \text { Charles }^{1} \text { And Patrick Ballard }}^{1}$
}

\begin{abstract}
This study deals with the existence and uniqueness of solutions to dynamical problems of finite freedom involving unilateral contact and Coulomb friction. In the frictionless case, it has been established [P. Ballard, Arch. Rational Mech. Anal. 154 (2000) 199-274] that the existence and uniqueness of a solution to the Cauchy problem can be proved under the assumption that the data are analytic, but not if they are assumed to be only of class $C^{\infty}$. Some years ago, this finding was extended [P. Ballard and S. Basseville, Math. Model. Numer. Anal. 39 (2005) 59-77] to the case where Coulomb friction is included in a model problem involving a single point particle. In the present paper, the existence and uniqueness of a solution to the Cauchy problem is proved in the case of a finite collection of particles in (possibly non-linear) interactions.
\end{abstract}

Mathematics Subject Classification. 70F40, 49J52, 34A60.

Received November 1st, 2012. Revised May 31, 2013.

Published online October 11, 2013.

\section{INTRODUCTION}

The question of the existence and uniqueness of a solution to dynamical problems involving unilateral contact and dry friction is still largely open.

These problems are of two kinds:

- continuum problems, on which very few results are available (for a recent survey, see [5]),

- finite freedom systems, such as a collection of rigid bodies or particles, or a system resulting from the spatial discretization of a continuum.

Different sets of equations are usually written to account for these two kinds of problems. In the case of (threedimensional, elastic or visco-elastic) continua, the usual elastodynamic equations are simply combined with boundary conditions of the Signorini type. In the case of finite freedom unilateral dynamical systems, such a set of equations is known to introduce some indetermination. The reason for this indetermination is that the bouncing of a real body against an obstacle is governed by the waves crossing the deformable body (and the obstacle). Since the dynamic theory of rigid bodies can not describe these waves, an indetermination arises, that

\footnotetext{
Keywords and phrases. Unilateral dynamics with friction, frictional dynamical contact problems, existence and uniqueness.

1 Laboratoire de Mécanique et d'Acoustique, CNRS, 31, chemin Joseph Aiguier, 13402 Marseille Cedex 20, France.

ballard@lma.cnrs-mrs.fr
} 
has to be solved by introducing an additional constitutive law: the impact constitutive law. One well-known example of an impact constitutive law is that based on Newton's famous restitution coefficient.

The well-posedness of the dynamics of discrete (synonymously, finite freedom) systems with unilateral constraints (without friction) was first investigated by Schatzman [14], who proved the existence of a solution using a penalization technique in the case of the elastic impact law (that is, with a restitution coefficient equal to 1). The latter author also gave a striking counter-example showing that, even in the cases where the data show regularity $C^{\infty}$, one cannot generally expect the solution to be unique. Percivale then commented in [13] that, in the case of the (necessarily frictionless) one degree-of-freedom problem with an external force depending only on time, the uniqueness of the solution can be restored by taking the external force to be an analytic function of time (instead of $C^{\infty}$ ). Schatzman [15] subsequently extended this finding about the uniqueness under analyticity assumptions, still in the context of the one degree-of-freedom problem, to the more general case where the external force is allowed to depend not only on time but also on the current position and velocity. However, her proof was specific to the one degree-of-freedom problem. A simpler proof was given by Ballard [2] who, then, extended these results to the general case involving an arbitrary number of degrees of freedom and unilateral constraints, but only in the case of frictionless situations.

The case of dry friction was first addressed by Monteiro Marques [7], in the case of a single smooth unilateral constraint and a completely inelastic impact law (that is, with a restitution coefficient equal to 0). Using the time-stepping algorithm introduced by Moreau $[9,10]$ (which is, roughly speaking, an adaptation of the implicit Euler scheme to the non-smooth situation under consideration) to build a sequence of approximants, Monteiro Marques succeeded in passing to the limit by extracting a subsequence using a compactness argument, resulting in a proof of existence of a solution. The issue of uniqueness was first addressed by Ballard and Basseville in [3] in the case of the simplest system involving unilateral contact and Coulomb friction - a system which was first introduced by Klarbring [6]. By extending Ballard's original method [2], these authors proved the uniqueness (under analyticity assumptions) of the solution also, in the case of this model problem involving dynamical unilateral contact and Coulomb friction. In the study by Ballard and Basseville, it was stated that the use of this method could be extended to more general situations than this model problem, but it was proposed to investigate in a future study how this could be achieved. The aim of the present paper is therefore to identify the more general situations in which existence and uniqueness can be proved using the method developed in [3].

In cases where a finite-degree-of-freedom system contains a rigid body that do not reduce to a point, examples of non-uniqueness or non-existence of solution to the Cauchy problem, which are known as Painlevé paradoxes, were exhibited by Painlevé more than one century ago [12]. The present analysis therefore focuses on the case of a finite collection of point particles. The case of rigid bodies that do not reduce to points requires, in our opinion, a re-visitation of the formulation of the evolution problem. This point is currently under investigation and will be extensively discussed in a forthcoming paper.

\section{Statement of the PRoblem And Results}

Let us take a finite collection of $n(n \geq 1)$ point particles in $\mathbb{R}^{d}(d=2,3)$. Some of these particles (for the sake of simplification, we will impose this constraint on all of them, without any loss of generality) have to remain on one side of a given obstacle associated with that particle. To simplify the writing slightly, it will be assumed in the first step, that each of these obstacles is straight, that is, it can be defined by a hyperplane. It is only in the second step (in Sect. 5) that we will deal with how the results can be extended to the case of possibly curved obstacles. For each particle, an affine orthonormal coordinate system, based on the corresponding straight obstacle, is introduced so that the position of the particle $i$ is described by:

$$
\mathbf{u}^{i}=\left(\begin{array}{l}
u_{\mathrm{n}}^{i} \in \mathbb{R} \\
\mathbf{u}_{\mathrm{t}}^{i} \in \mathbb{R}^{d-1}
\end{array}\right) \in \mathbb{R}^{d},
$$




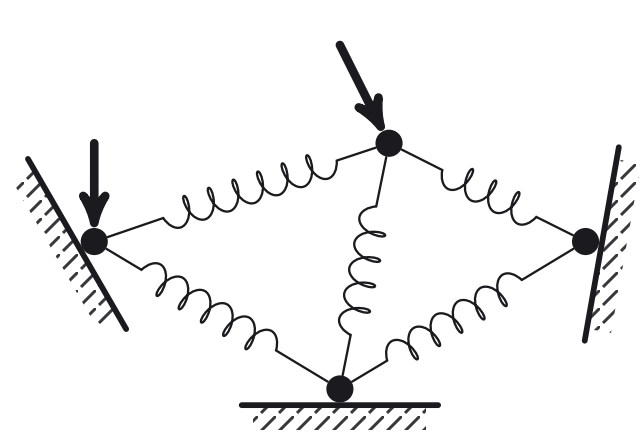

(a) straight obstacles.

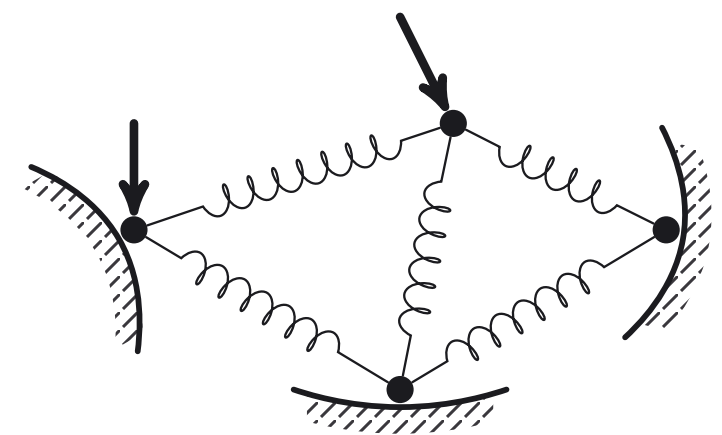

(b) curved obstacles.

FiguRE 1. Geometry of the problem.

and the unilateral constraint reduces to $u_{\mathrm{n}}^{i} \leq 0$. A configuration of the system of particles will be described by:

$$
\mathbf{U}=\left(\begin{array}{c}
\mathbf{u}^{1} \in \mathbb{R}^{d} \\
\vdots \\
\mathbf{u}^{n} \in \mathbb{R}^{d}
\end{array}\right) \in \mathbb{R}^{n d}
$$

We take:

$$
\mathbf{U}_{\mathrm{n}}=\left(\begin{array}{c}
u_{\mathrm{n}}^{1} \in \mathbb{R} \\
\vdots \\
u_{\mathrm{n}}^{n} \in \mathbb{R}
\end{array}\right) \in \mathbb{R}^{n},
$$

to denote the vector formed by the normal components of each particle. A similar vector $\mathbf{U}_{\mathbf{t}} \in \mathbb{R}^{n(d-1)}$ formed by the tangential components will also be used below.

It is now proposed to formulate a Cauchy problem associated with the dynamics of this system of particles subjected to internal and external forces and unilateral contact with Coulomb friction with respect to the obstacles. This requires introducing a reaction force $\mathbf{r}^{i} \in \mathbb{R}^{d}$ for the particle having the index $i$. The normal component of this reaction force, which will be denoted by $r_{\mathrm{n}}^{i}$, will be required to be non-positive (non-adhesive unilateral contact). The reaction force at work in the system will be denoted by $\mathbf{R} \in \mathbb{R}^{\text {nd }}$ (of normal component $\mathbf{R}_{\mathrm{n}} \in \mathbb{R}^{n}$ ) and is built with the $\mathbf{r}^{i}$ in the same way as $\mathbf{U}$ is based on the $\mathbf{u}^{i}$. Given an arbitrary time interval $I$, a motion of the system will be described by a function $\mathbf{U}(t)(t \in I)$. Due to the obstacles, we must expect velocity jumps, that is, impacts. Hence, the acceleration $\ddot{\mathbf{U}}$ will have to be understood in the sense of distributions and not in the classical sense. Since the distribution $\mathbf{R}: I \rightarrow \mathbb{R}^{\text {nd }}$ is required to take values in a cone, it classically entails that it is a measure on $I$ with values in $\mathbb{R}^{\text {nd }}$. Hence, the acceleration will be a measure (notation $\ddot{\mathbf{U}} \in \mathcal{M}\left(I, \mathbb{R}^{n d}\right)$ ) and the search for the motion will be conducted in the space $M M A\left(I, \mathbb{R}^{n d}\right)$, consisting of all the distributions on $I$ whose twice-derivative is a measure. This space was first introduced by Schatzman in [14]. It contains only continuous functions, having left and right derivatives $\dot{\mathbf{U}}^{-}(t)$ and $\dot{\mathbf{U}}^{+}(t)$ (in the classical sense) at each $t$ in the interior of $I$. The functions $\dot{\mathbf{U}}^{-}(t)$ and $\dot{\mathbf{U}}^{+}(t)$ are functions with locally bounded variation. Given an arbitrary $T>0$, the Cauchy problem can now be formulated in line with Moreau $[8,10]$. 
Problem $\mathcal{P}_{\mathbf{u}}$. Find $\mathbf{U} \in \operatorname{MMA}\left([0, T] ; \mathbb{R}^{n d}\right)$ and $\mathbf{R} \in \mathcal{M}\left([0, T] ; \mathbb{R}^{n d}\right)$ such that:

- $\mathbf{U}(0)=\mathbf{U}_{0} \quad ; \quad \dot{\mathbf{U}}^{+}(0)=\mathbf{V}_{0}$

- $\ddot{\mathbf{U}}=\mathbf{F}(t, \mathbf{U}, \dot{\mathbf{U}})+\mathbf{R}, \quad$ in $[0, T]$

- $\forall i \in\{1,2, \ldots, n\}, \quad \forall t \in] 0, T[$,

$$
u_{\mathrm{n}}^{i} \leq 0, \quad r_{\mathrm{n}}^{i} \leq 0, \quad u_{\mathrm{n}}^{i} r_{\mathrm{n}}^{i}=0
$$

- $\forall i \in\{1,2, \ldots, n\}, \quad \forall \mathbf{v} \in C^{0}\left([0, T] ; \mathbb{R}^{d-1}\right)$,

$$
\int_{[0, T]}\left[\mathbf{r}_{\mathrm{t}}^{i} \cdot\left(\mathbf{v}-\dot{\mathbf{u}}_{\mathrm{t}}^{i+}\right)-\mu_{i} r_{\mathrm{n}}^{i}\left(\|\mathbf{v}\|-\left\|\dot{\mathbf{u}}_{\mathrm{t}}^{i+}\right\|\right)\right] \geq 0
$$

- $\forall i \in\{1,2, \ldots, n\}, \quad \forall t \in] 0, T[$,

$$
u_{\mathrm{n}}^{i}(t)=0 \quad \Longrightarrow \quad \dot{u}_{\mathrm{n}}^{i+}(t)=-e_{i} \dot{u}_{\mathrm{n}}^{i-}(t),
$$

(initial condition),

(motion equation),

(unilateral contact),

where the data are as follows.

- The initial condition $\left(\mathbf{U}_{0}, \mathbf{V}_{0}\right) \in \mathbb{R}^{n d} \times \mathbb{R}^{n d}$ is assumed to be compatible with the unilateral constraint:

$$
\mathbf{U}_{0 \mathrm{n}} \leq 0 \quad \text { and } \quad u_{0 \mathrm{n}}^{i}=0 \quad \Longrightarrow \quad v_{0 \mathrm{n}}^{i} \leq 0,
$$

where, by convention, $\mathbf{v} \leq 0$, for $\mathbf{v} \in \mathbb{R}^{n}$, means that all its components are non-positive.

- The force mapping $\mathbf{F}(t, \mathbf{U}, \dot{\mathbf{U}})$ is a given mapping $\mathbf{F}:[0, T] \times \mathbb{R}^{n d} \times \mathbb{R}^{n d} \rightarrow \mathbb{R}^{\text {nd }}$. The force acting on the particle having the index $i$ will be denoted by $\mathbf{f}^{i}(t, \mathbf{U}, \dot{\mathbf{U}})$, so that $\mathbf{F}$ is obtained from the $\mathbf{f}^{i}$ in the same way as $\mathbf{U}$ is based on the $\mathbf{u}^{i}$.

- The $\mu_{i} \geq 0$ are the friction coefficients of each particle, and the $e_{i} \in[0,1]$ are the restitution coefficients.

A weak formulation for the Coulomb friction law has been used here because the reaction force is generally a measure. In cases where this measure reduces to some continuous function, it can easily be checked that this weak formulation is equivalent to the usual pointwise formulation. The following theorem will be proved below.

Theorem 2.1. Let us assume that the function $\mathbf{F}:[0, T] \times \mathbb{R}^{n d} \times \mathbb{R}^{n d} \rightarrow \mathbb{R}^{\text {nd }}$ satisfies the following hypotheses:

(i) the function $(\mathbf{U}, \mathbf{V}) \mapsto \mathbf{F}(t, \mathbf{U}, \mathbf{V})$ satisfies a Lipschitz condition of modulus $\kappa \geq 0$, for all $t \in[0, T]$ :

$$
\begin{aligned}
& \forall t \in[0, T], \quad \forall\left(\mathbf{U}_{1}, \mathbf{V}_{1}\right),\left(\mathbf{U}_{2}, \mathbf{V}_{2}\right) \in \mathbb{R}^{n d} \times \mathbb{R}^{n d}, \\
&\left\|\mathbf{F}\left(t, \mathbf{U}_{1}, \mathbf{V}_{1}\right)-\mathbf{F}\left(t, \mathbf{U}_{2}, \mathbf{V}_{2}\right)\right\| \leq \kappa\left\|\left(\mathbf{U}_{1}, \mathbf{V}_{1}\right)-\left(\mathbf{U}_{2}, \mathbf{V}_{2}\right)\right\|,
\end{aligned}
$$

where $\|\cdot\|$ stands for the Euclidean norm in $\mathbb{R}^{\text {nd }} \times \mathbb{R}^{\text {nd }}$;

(ii) the function $(t, \mathbf{U}, \mathbf{V}) \mapsto \mathbf{F}(t, \mathbf{U}, \mathbf{V})$ is analytic;

(iii) each $\mathbf{f}^{i}$ does not depend on the $\dot{\mathbf{u}}^{j}$ when $j \neq i$.

Then, problem $\mathcal{P}_{\mathbf{u}}$ has one and only one solution.

The hypotheses adopted in Theorem 2.1 do not all have the same status. Hypothesis (i) is simply used for convenience and is not essential. Dropping it would permit the solution to blow up at a finite time, so that the conclusion in Theorem 2.1 would have to be replaced by: there exists one and only one maximal solution (in the sense that any other solution is a restriction of that maximal solution to a smaller time interval). Hypothesis (ii) was adopted because even in the frictionless situations, multiple solutions can be encountered with $\mathbf{F} \in C^{\infty}$. Hypothesis (ii) was also adopted in the preliminary analysis of a model problem in [3]. Hypothesis (iii) is something else. It turns out that the strategy on which the proof in [3] was based can be used to deal with 
cases where the force mapping $\mathbf{F}(t, \mathbf{U})$ depends arbitrarily on the configuration but not on the velocity. We were surprised to discover that this strategy does not seem to be valid in cases where the force mapping $\mathbf{F}(t, \mathbf{U}, \dot{\mathbf{U}})$ depends arbitrarily on the velocity $\dot{\mathbf{U}}$, and we have no idea so far how to extend the result to that case, or even if it is true. In concrete terms, hypothesis (iii) excludes viscous interactions between particles undergoing unilateral constraints. This is a severe restriction which requires better understanding in the future. Note however, that for a system consisting of a single particle, hypothesis (iii) is automatically satisfied and is no restriction at all.

The strategy used to prove Theorem 2.1 will be the same as that used in [3]. First, the unilateral constraints will be frozen into bilateral constraints. Due to Coulomb friction, the bilateral problem is not governed by an ordinary differential equation as in the frictionless situation studied in [2]. In Section 3.1, the bilateral problem is expressed in the form of a differential inclusion which is solved in Section 3.2 using standard monotonicity techniques as in [3]. It is then proved in Section 3.3 that the restriction of the solution to some right-neighborhood of the time origin is analytic, as long as the external force is analytic. The analysis of the bilateral problem, as performed in Section 3, is used in Section 4.1 to build a local analytic solution to the unilateral problem with an analytic external force. To ensure well-posedness of the unilateral problem, it then suffices to prove that there cannot exist any other local solution in MMA, which differs from the local analytic one. This is done in Section 4.2 by adapting Ballard's strategy [2] to the situation under consideration.

Theorem 2.1 extends to those cases where the obstacles may be curved instead of being straight. Details about how this extension is achieved are given in Section 5 .

\section{The Bilateral PROBlem}

In this section, we study the simpler situation where some of the unilateral constraints are frozen into bilateral constraints and the other unilateral constraints are simply dropped.

\subsection{Formulation of the bilateral problem}

We are given two sets $\mathcal{B}, \mathcal{I}$ of indices such that $\mathcal{B} \cap \mathcal{I}=\varnothing$ and $\mathcal{B} \cup \mathcal{I}=\{1,2 \ldots, n\}$, and the unilateral contact condition in problem $\mathcal{P}_{\mathbf{u}}$ is replaced by:

$$
\forall i \in \mathcal{B}, \quad \mathbf{u}_{\mathrm{n}}^{i} \equiv 0, \quad \forall i \in \mathcal{I}, \quad \mathbf{r}_{\mathrm{n}}^{i} \equiv 0 .
$$

We shall therefore consider the following evolution problem.

Problem $\mathcal{P}_{\mathbf{b}}$. Find $\mathbf{U} \in M M A\left([0, T] ; \mathbb{R}^{n d}\right)$ and $\mathbf{R} \in \mathcal{M}\left([0, T] ; \mathbb{R}^{n d}\right)$ such that:

- $\mathbf{U}(0)=\mathbf{U}_{0} \quad ; \quad \dot{\mathbf{U}}^{+}(0)=\mathbf{V}_{0}$

- $\ddot{\mathbf{U}}=\mathbf{F}(t, \mathbf{U}, \dot{\mathbf{U}})+\mathbf{R}, \quad$ in $[0, T]$

- $\forall i \in \mathcal{B}, \quad \mathbf{u}_{\mathrm{n}}^{i} \equiv 0$,

- $\forall i \in \mathcal{I}, \quad \mathbf{r}_{\mathrm{n}}^{i} \equiv 0$,

- $\forall i \in\{1,2, \ldots, n\}, \quad \forall \mathbf{v} \in C^{0}\left([0, T] ; \mathbb{R}^{d-1}\right)$,

$$
\int_{[0, T]}\left[\mathbf{r}_{\mathrm{t}}^{i} \cdot\left(\mathbf{v}-\dot{\mathbf{u}}_{\mathrm{t}}^{i+}\right)+\mu_{i}\left|r_{\mathrm{n}}^{i}\right|\left(\|\mathbf{v}\|-\left\|\dot{\mathbf{u}}_{\mathrm{t}}^{i+}\right\|\right)\right] \geq 0,
$$

(initial condition), (motion equation), (bilateral constraints), (inactive constraints),

(Coulomb friction),

where the initial condition $\left(\mathbf{U}_{0}, \mathbf{V}_{0}\right) \in \mathbb{R}^{n d} \times \mathbb{R}^{n d}$ is assumed to be compatible with the bilateral constraint:

$$
\forall i \in \mathcal{B}, \quad u_{0 \mathrm{n}}^{i}=0 \quad \text { and } \quad v_{0 \mathrm{n}}^{i}=0 .
$$

From now on, the function $\mathbf{F}$ is supposed to satisfy both condition (i) of page 4 and:

(ii') For all $(\mathbf{U}, \mathbf{V}) \in \mathbb{R}^{n d} \times \mathbb{R}^{n d}$, the function $t \mapsto \mathbf{F}(t, \mathbf{U}, \mathbf{V})$ is integrable over $[0, T]$. 
These hypotheses are weaker than those in Theorem 2.1. If $\mathbf{U}$ denotes an arbitrary solution of problem $\mathcal{P}_{\mathbf{b}}$, then $\mathbf{U}$ is absolutely continuous and $\dot{\mathbf{U}}$ has bounded variation, so that they are both integrable functions. Hence, the hypotheses made about $\mathbf{F}$ entail that the (almost everywhere defined) function $t \mapsto \mathbf{F}_{\mathrm{n}}(t, \mathbf{U}(t), \dot{\mathbf{U}}(t)$ ) is integrable. Furthermore the normal components of the motion equation read as follows:

$$
\ddot{\mathbf{U}}_{\mathrm{n}}=\mathbf{F}_{\mathrm{n}}(t, \mathbf{U}, \dot{\mathbf{U}})+\mathbf{R}_{\mathrm{n}},
$$

so that:

$$
\begin{array}{ll}
r_{\mathrm{n}}^{i}=-f_{\mathrm{n}}^{i}(t, \mathbf{U}, \dot{\mathbf{U}}), & \text { if } i \in \mathcal{B}, \\
r_{\mathrm{n}}^{i}=0, & \text { if } i \in \mathcal{I} .
\end{array}
$$

Therefore, the measures $r_{\mathrm{n}}^{i}$ are absolutely continuous with respect to the Lebesgue measure. But the Coulomb friction law entails the following inequality between the absolute value measures:

$$
\forall i \in\{1,2, \ldots, n\}, \quad\left\|\mathbf{r}_{\mathrm{t}}^{i}\right\| \leq \mu_{i}\left|r_{\mathrm{n}}^{i}\right|,
$$

which shows that the $\mathbf{r}_{\mathrm{t}}^{i}$ are also absolutely continuous with respect to the Lebesgue measure, that is, they are integrable functions. Hence, any solution $(\mathbf{U}, \mathbf{R})$ of problem $\mathcal{P}_{\mathbf{b}}$ must actually belong to $W^{2,1} \times L^{1}$.

Next, denote by $\mathcal{R}(t, \mathbf{U}, \dot{\mathbf{U}})$ the vector in $\mathbb{R}^{n}$ with components:

$$
\mathcal{R}^{i}(t, \mathbf{U}, \dot{\mathbf{U}})=\mid \begin{array}{ll}
\mu_{i} f_{\mathrm{n}}^{i}(t, \mathbf{U}, \dot{\mathbf{U}}), & \text { if } i \in \mathcal{B}, \\
0, & \text { if } i \in \mathcal{I} .
\end{array}
$$

The vector $\mathcal{R}(t, \mathbf{U}, \dot{\mathbf{U}})$ contains the friction thresholds for each particle. Note that the regularity hypotheses adopted about the function $\mathbf{F}$ also hold true for the function $\mathcal{R}(t, \mathbf{U}, \dot{\mathbf{U}})$. Let $\bar{B}$ the closed unit ball in $\mathbb{R}^{d-1}$. The support function of a non-empty closed convex set $K$ will be denoted by $S_{K}$ and its sub-differential by $\partial S_{K}$. With these notations, the following differential inclusion:

$$
\ddot{\mathbf{u}}_{\mathrm{t}}^{i}(t)-\mathbf{f}_{\mathrm{t}}^{i}(t, \mathbf{U}(t), \dot{\mathbf{U}}(t)) \in \partial S_{\left|\mathcal{R}^{i}(t, \mathbf{U}(t), \dot{\mathbf{U}}(t))\right| \bar{B}}\left[-\dot{\mathbf{u}}_{\mathrm{t}}^{i}(t)\right]
$$

holds true, for almost all $t \in[0, T]$ and all $i \in\{1,2 \ldots, n\}$. For $\mathcal{R} \in \mathbb{R}^{n}$, we will use the short-hand notation:

$$
|\mathcal{R}| \cdot \bar{B} \stackrel{\text { def }}{=} \bigotimes_{i=1}^{n}\left|\mathcal{R}^{i}\right| \bar{B} \subset \mathbb{R}^{n(d-1)},
$$

where the $\bigotimes$ stands for the Cartesian product. Hence, for $\mathcal{R} \in \mathbb{R}^{n}$ and $\mathbf{X} \in \mathbb{R}^{n(d-1)}$, one has the identity:

$$
\partial S_{|\mathcal{R}| \cdot \bar{B}}[\mathbf{X}]=\bigotimes_{i=1}^{n} \partial S_{\left|\mathcal{R}^{i}\right| \bar{B}}\left[\mathbf{X}^{i}\right]
$$

and therefore, the following differential inclusion:

$$
\ddot{\mathbf{U}}_{\mathrm{t}}(t)-\mathbf{F}_{\mathrm{t}}(t, \mathbf{U}(t), \dot{\mathbf{U}}(t)) \in \partial S_{|\mathcal{R}(t, \mathbf{U}(t), \dot{\mathbf{U}}(t))| \cdot \bar{B}}\left[-\dot{\mathbf{U}}_{\mathrm{t}}(t)\right],
$$

holds true, for almost all $t \in[0, T]$. For the normal components, note that:

$$
\begin{aligned}
& \ddot{u}_{\mathrm{n}}^{i}=0, \text { if } i \in \mathcal{B}, \\
& r_{\mathrm{n}}^{i}=0, \text { if } i \in \mathcal{I},
\end{aligned}
$$

so that, defining a function $\mathcal{F}(t, \mathbf{U}, \mathbf{V})$ such that $\mathcal{F}_{\mathrm{t}} \equiv \mathbf{F}_{\mathrm{t}}$ and:

$$
\begin{aligned}
& \mathcal{F}_{\mathrm{n}}^{i} \equiv 0, \quad i \in \mathcal{B}, \\
& \mathcal{F}_{\mathrm{n}}^{i} \equiv \mathbf{f}_{\mathrm{n}}^{i}, i \in \mathcal{I},
\end{aligned}
$$


we obtain the following differential inclusion obeyed by $\mathbf{U}$ :

$$
\ddot{\mathbf{U}}(t)-\mathcal{F}(t, \mathbf{U}(t), \dot{\mathbf{U}}(t)) \in \partial S_{|\mathcal{R}(t, \mathbf{U}(t), \dot{\mathbf{U}}(t))| \cdot \bar{C}}[-\dot{\mathbf{U}}(t)],
$$

with the notation $\bar{C} \stackrel{\text { def }}{=}\{0\} \times \bar{B} \subset \mathbb{R}^{d}$. Finally, problem $\mathcal{P}_{\mathbf{b}}$ can be handled using the following simpler but equivalent formulation.

Problem $\mathcal{P}_{\mathbf{b}}$. Find $\mathbf{U} \in W^{2,1}\left(0, T ; \mathbb{R}^{n d}\right)$ such that:

$$
\begin{aligned}
& \text { - } \mathbf{U}(0)=\mathbf{U}_{0} \quad ; \quad \dot{\mathbf{U}}(0)=\mathbf{V}_{0} \quad \text { (initial condition), } \\
& \text { - } \ddot{\mathbf{U}}(t)-\mathcal{F}(t, \mathbf{U}(t), \dot{\mathbf{U}}(t)) \in \partial S_{|\mathcal{R}(t, \mathbf{U}(t), \dot{\mathbf{U}}(t))| \cdot \bar{C}}[-\dot{\mathbf{U}}(t)], \text { for a.a. } t \text {, }
\end{aligned}
$$

where the functions $\mathcal{F}(t, \mathbf{U}, \mathbf{V})$ and $\mathcal{R}(t, \mathbf{U}, \mathbf{V})$ satisfy hypotheses (i) and (ii') adopted about $\mathbf{F}(t, \mathbf{U}, \mathbf{V})$.

\subsection{Existence and uniqueness of a solution to the bilateral problem}

This section is devoted to proving the following proposition.

Proposition 3.1. Problem $\mathcal{P}_{\mathbf{b}}$ admits a unique solution $\mathbf{U} \in W^{2,1}\left(0, T ; \mathbb{R}^{\text {nd }}\right)$.

Proof.

Step 1. Proposition 3.1 holds true in the particular case where the function $\mathcal{R}$ is constant.

In the case where the function $\mathcal{R}$ is constant and the function $\mathcal{F}$ is simply an integrable function of time, Proposition 3.1 holds true by virtue of proposition 3.4, p. 69 of [4]. In the case where $\mathcal{F}$ also depends on (U, $\mathbf{U})$, the method of successive approximations will be used. Let $\left(\mathbf{V}^{k}\right)$ be the sequence of functions in $W^{1,1}\left(0, T ; \mathbb{R}^{\text {nd }}\right)$ defined by $\mathbf{V}^{0} \equiv \mathbf{V}_{0}$ and the following induction. If $\mathbf{V}^{k}$ is given, standard theorems on differential inclusions associated with the subdifferential of a lower semi-continuous function (see, for example, Prop. 3.4, p. 69 of [4]) provide a unique solution $\mathbf{V}^{k+1} \in W^{1,1}\left(0, T ; \mathbb{R}^{n d}\right)$ of the evolution problem:

- $\mathbf{V}^{k+1}(0)=\mathbf{V}_{0}$,

- $\dot{\mathbf{V}}^{k+1}(t)-\mathcal{F}\left(t, \mathbf{U}_{0}+\int_{0}^{t} \mathbf{V}^{k}, \mathbf{V}^{k}(t)\right) \in \partial S_{|\mathcal{R}| \cdot \bar{C}}\left[-\mathbf{V}^{k+1}(t)\right], \quad$ for a.a. $t$.

We are now going to prove the convergence of the sequence $\left(\mathbf{V}^{k}\right)$ and establish that the limit solves the expected evolution problem. Based on the monotonicity of the sub-differential, we have:

$$
\left[\mathbf{V}^{k+1}(t)-\mathbf{V}^{k}(t)\right] \cdot\left[\dot{\mathbf{V}}^{k+1}(t)-\dot{\mathbf{V}}^{k}(t)-\mathcal{F}\left(t, \mathbf{U}_{0}+\int_{0}^{t} \mathbf{V}^{k}, \mathbf{V}^{k}(t)\right)+\mathcal{F}\left(t, \mathbf{U}_{0}+\int_{0}^{t} \mathbf{V}^{k-1}, \mathbf{V}^{k-1}(t)\right)\right] \leq 0,
$$

for almost all $t \in[0, T]$. Integrating:

$\frac{1}{2}\left\|\mathbf{V}^{k+1}(t)-\mathbf{V}^{k}(t)\right\|^{2} \leq \int_{0}^{t}\left\|\mathbf{V}^{k+1}(s)-\mathbf{V}^{k}(s)\right\| .\left\|\mathcal{F}\left(s, \mathbf{U}_{0}+\int_{0}^{s} \mathbf{V}^{k}, \mathbf{V}^{k}(s)\right)-\mathcal{F}\left(s, \mathbf{U}_{0}+\int_{0}^{s} \mathbf{V}^{k-1}, \mathbf{V}^{k-1}(s)\right)\right\| \mathrm{d} s$,

and, from Lemma A. $5^{2}$, p. 157 of [4]:

$$
\forall t \in[0, T], \quad\left\|\mathbf{V}^{k+1}(t)-\mathbf{V}^{k}(t)\right\| \leq \int_{0}^{t}\left\|\mathcal{F}\left(s, \mathbf{U}_{0}+\int_{0}^{s} \mathbf{V}^{k}, \mathbf{V}^{k}(s)\right)-\mathcal{F}\left(s, \mathbf{U}_{0}+\int_{0}^{s} \mathbf{V}^{k-1}, \mathbf{V}^{k-1}(s)\right)\right\| \mathrm{d} s .
$$

\footnotetext{
${ }^{2}$ This lemma is the following assertion. Let $a$ be nonnegative real constant, $m$ be a nonnegative locally integrable function and $\phi$ a continuous function satisfying:

$$
\forall t \geq 0, \quad \frac{1}{2} \phi^{2}(t) \leq \frac{1}{2} a^{2}+\int_{0}^{t} m(s) \phi(s) \mathrm{d} s .
$$
}

Then:

$$
\forall t \geq 0, \quad|\phi(t)| \leq a+\int_{0}^{t} m(s) \phi(s) \mathrm{d} s
$$


Using the assumption that $\mathcal{F}$ is Lipschitz-continuous with respect to $(\mathbf{U}, \mathbf{V})$, we obtain:

$$
\begin{aligned}
\left\|\mathbf{V}^{k+1}(t)-\mathbf{V}^{k}(t)\right\| & \leq \kappa \int_{0}^{t}\left(\left\|\mathbf{V}^{k}(s)-\mathbf{V}^{k-1}(s)\right\|+\int_{0}^{s}\left\|\mathbf{V}^{k}-\mathbf{V}^{k-1}\right\|\right) \mathrm{d} s, \\
& \leq \kappa(1+T) \int_{0}^{t}\left\|\mathbf{V}^{k}(s)-\mathbf{V}^{k-1}(s)\right\| \mathrm{d} s .
\end{aligned}
$$

By induction, we obtain:

$$
\forall k \in \mathbb{N}, \quad \forall t \in[0, T], \quad\left\|\mathbf{V}^{k+1}(t)-\mathbf{V}^{k}(t)\right\| \leq \frac{[\kappa t(1+T)]^{k}}{k !}\left\|\mathbf{V}^{1}-\mathbf{V}^{0}\right\|_{C^{0}},
$$

which entails that, since the sequence $\left(\mathbf{V}^{k}\right)$ is a Cauchy sequence, it converges uniformly in $[0, T]$ towards some limit $\mathbf{V} \in C^{0}\left([0, T] ; \mathbb{R}^{n d}\right)$. Now, let $\mathbf{W} \in W^{1,1}\left(0, T ; \mathbb{R}^{n d}\right)$ be the unique solution of the following evolution problem:

- $\mathbf{W}(0)=\mathbf{V}_{0}$,

- $\dot{\mathbf{W}}(t)-\mathcal{F}\left(t, \mathbf{U}_{0}+\int_{0}^{t} \mathbf{V}, \mathbf{V}(t)\right) \in \partial S_{|\mathcal{R}| \cdot \bar{C}}[-\mathbf{W}(t)], \quad$ for a.a. $t$.

Taking the difference between the latter differential inclusion and that defining $\mathbf{V}^{k+1}$, multiplying by $\mathbf{W}-\mathbf{V}^{k+1}$, and given the monotonicity of the sub-differential and then Lemma A.5, p. 157 of [4], we obtain:

$$
\forall k \in \mathbb{N}, \quad \forall t \in[0, T], \quad\left\|\mathbf{W}(t)-\mathbf{V}^{k+1}(t)\right\| \leq \kappa(1+T) \int_{0}^{t}\left\|\mathbf{V}-\mathbf{V}^{k}\right\|
$$

Taking the limit $k \rightarrow \infty$, it can be seen that the sequence $\left(\mathbf{V}^{k}\right)$ converges uniformly in $[0, T]$ towards $\mathbf{W}=\mathbf{V} \in$ $W^{1,1}\left(0, T ; \mathbb{R}^{n d}\right)$, so that:

$$
\begin{aligned}
& \text { - } \mathbf{V}(0)=\mathbf{V}_{0}, \\
& \text { - } \dot{\mathbf{V}}(t)-\mathcal{F}\left(t, \mathbf{U}_{0}+\int_{0}^{t} \mathbf{V}, \mathbf{V}(t)\right) \in \partial S_{|\mathcal{R}| \cdot \bar{C}}[-\mathbf{V}(t)], \quad \text { for a.a. } t,
\end{aligned}
$$

and the function $\mathbf{U}(t) \stackrel{\text { def }}{=} \mathbf{U}_{0}+\int_{0}^{t} \mathbf{V}$ is a solution of the evolution problem under consideration.

To establish that this is the only solution, take another one, say $\mathbf{U}^{\prime}$. Using the same algebra as above, based on the monotonicity of the sub-differential, we obtain:

$$
\forall t \in[0, T], \quad\left\|\dot{\mathbf{U}}(t)-\dot{\mathbf{U}}^{\prime}(t)\right\| \leq \kappa(1+T) \int_{0}^{t}\left\|\dot{\mathbf{U}}-\dot{\mathbf{U}}^{\prime}\right\|
$$

and Gronwall's lemma yields $\mathbf{U} \equiv \mathbf{U}^{\prime}$.

Step 2. Proposition 3.1 holds true in the particular case where the function $\mathcal{R}$ is simply an integrable function of time.

In this step, it is proposed to extend the result proved in step 1 for the case where $\mathcal{R}$ is a constant function to the case where it is an integrable function of time $t$ alone. Note that since the proof of uniqueness part of step 1 is also valid in this extended case, only the existence of a solution has to be proved.

First, we take a sequence $\left(\mathcal{R}^{k}\right)$ of piecewise constant functions that converges strongly in $L^{1}\left(0, T ; \mathbb{R}^{n}\right)$ towards the integrable function $\mathcal{R}$. The sequence $\left(\mathcal{R}^{k}\right)$ can be chosen so that all the components with index $i \in \mathcal{I}$ vanish. Based on step 1 , there exists a sequence $\left(\mathbf{U}^{k}\right)$ in $W^{2,1}\left(0, T ; \mathbb{R}^{n d}\right)$ such that:

$$
\begin{array}{ll}
\text { - } \mathbf{U}^{k}(0)=\mathbf{U}_{0} \quad ; \quad \dot{\mathbf{U}}^{k}(0)=\mathbf{V}_{0} \quad \text { (initial condition), } \\
\text { - } \ddot{\mathbf{U}}^{k}(t)-\mathcal{F}\left(t, \mathbf{U}^{k}(t), \dot{\mathbf{U}}^{k}(t)\right) \in \partial S_{\left|\mathcal{R}^{k}(t)\right| \cdot \bar{C}}\left[-\dot{\mathbf{U}}^{k}(t)\right], & \text { for a.a. } t .
\end{array}
$$


From the definition of the sub-differential, for $f \in \partial S_{r \bar{B}}[x]$ and $f^{\prime} \in \partial S_{r^{\prime}}\left[x^{\prime}\right]$ :

$$
\begin{aligned}
& r\left\|x^{\prime}\right\| \geq r\|x\|+f \cdot\left(x^{\prime}-x\right), \\
& r^{\prime}\|x\| \geq r^{\prime}\left\|x^{\prime}\right\|+f^{\prime} \cdot\left(x-x^{\prime}\right),
\end{aligned}
$$

and summing up, we obtain:

$$
\left(f^{\prime}-f\right) \cdot\left(x^{\prime}-x\right) \geq\left(r^{\prime}-r\right)\left(\left\|x^{\prime}\right\|-\|x\|\right)
$$

Hence:

$$
\begin{aligned}
\left(\ddot{\mathbf{U}}^{p+q}(t)\right. & \left.-\ddot{\mathbf{U}}^{p}(t)\right) \cdot\left(\dot{\mathbf{U}}^{p+q}(t)-\dot{\mathbf{U}}^{p}(t)-\mathcal{F}\left(t, \mathbf{U}^{p+q}(t), \dot{\mathbf{U}}^{p+q}(t)\right)+\mathcal{F}\left(t, \mathbf{U}^{p}(t), \dot{\mathbf{U}}^{p}(t)\right)\right) \\
& =\left(\ddot{\mathbf{U}}_{\mathrm{t}}^{p+q}(t)-\ddot{\mathbf{U}}_{\mathrm{t}}^{p}(t)\right) \cdot\left(\dot{\mathbf{U}}_{\mathrm{t}}^{p+q}(t)-\dot{\mathbf{U}}_{\mathrm{t}}^{p}(t)-\mathcal{F}_{\mathrm{t}}\left(t, \mathbf{U}^{p+q}(t), \dot{\mathbf{U}}^{p+q}(t)\right)+\mathcal{F}_{\mathrm{t}}\left(t, \mathbf{U}^{p}(t), \dot{\mathbf{U}}^{p}(t)\right)\right), \\
& \leq-\sum_{i=1}^{n}\left(\left|\mathcal{R}^{p+q, i}(t)\right|-\left|\mathcal{R}^{p, i}(t)\right|\right)\left(\left\|\dot{\mathbf{u}}^{p+q, i}(t)\right\|-\left\|\dot{\mathbf{u}}^{p, i}(t)\right\|\right), \\
& \leq\left[\sum_{i=1}^{n}\left|\mathcal{R}^{p+q, i}(t)-\mathcal{R}^{p, i}(t)\right|\right]\left\|\dot{\mathbf{U}}^{p+q}(t)-\dot{\mathbf{U}}^{p}(t)\right\|,
\end{aligned}
$$

where $\mathcal{R}^{p, i}$ denotes the $i$ th component of $\mathcal{R}^{p}$. Integrating and then, using the Lipschitz property for $\mathcal{F}$ and Lemma A.5, p. 157 of [4], we obtain:

$$
\begin{aligned}
\left\|\dot{\mathbf{U}}^{p+q}(t)-\dot{\mathbf{U}}^{p}(t)\right\| & \leq \int_{0}^{t} \sum_{i=1}^{n}\left|\mathcal{R}^{p+q, i}-\mathcal{R}^{p, i}\right|+\kappa \int_{0}^{t}\left\|\mathbf{U}^{p+q}-\mathbf{U}^{p}\right\|+\kappa \int_{0}^{t}\left\|\dot{\mathbf{U}}^{p+q}-\dot{\mathbf{U}}^{p}\right\|, \\
& \leq \int_{0}^{t} \sum_{i=1}^{n}\left|\mathcal{R}^{p+q, i}-\mathcal{R}^{p, i}\right|+\kappa(1+T) \int_{0}^{t}\left\|\dot{\mathbf{U}}^{p+q}-\dot{\mathbf{U}}^{p}\right\|
\end{aligned}
$$

Based on the Gronwall-Bellman lemma (Lem. A.4, p. 156 of [4]):

$$
\forall t \in[0, T], \quad\left\|\dot{\mathbf{U}}^{p+q}(t)-\dot{\mathbf{U}}^{p}(t)\right\| \leq \mathrm{e}^{\kappa T(1+T)} \int_{0}^{T}\left[\sum_{i=1}^{n}\left|\mathcal{R}^{p+q, i}-\mathcal{R}^{p, i}\right|\right] .
$$

Hence, the sequence $\left(\dot{\mathbf{U}}^{k}\right)$ is Cauchy in the Banach space $C^{0}\left([0, T], \mathbb{R}^{n d}\right)$ and therefore converges in that space towards some limit $\mathbf{V} \in C^{0}$. Defining $\mathbf{U}=\mathbf{U}_{0}+\int_{0}^{t} \mathbf{V}$, it can be readily checked that $\left(\mathbf{U}^{k}\right)$ converges uniformly on $[0, T]$ towards $\mathbf{U}$ and that $\dot{\mathbf{U}}=\mathbf{V}$.

We shall now prove that $\mathbf{U} \in W^{2,1}$ and is the expected solution of the evolution problem under consideration. Take $0 \leq t_{1} \leq s \leq t_{2} \leq T$. Based on the definition of the sub-differential:

$$
\begin{aligned}
\left(\dot{\mathbf{U}}^{k}(s)-\dot{\mathbf{U}}^{k}\left(t_{1}\right)\right) \cdot\left(\ddot{\mathbf{U}}^{k}(s)-\mathcal{F}\left(s, \mathbf{U}^{k}(s), \dot{\mathbf{U}}^{k}(s)\right)\right) & \leq \sum_{i=1}^{n}\left|\mathcal{R}^{k, i}(s)\right|\left(\left\|\dot{\mathbf{u}}^{k, i}\left(t_{1}\right)\right\|-\left\|\dot{\mathbf{u}}^{k, i}(s)\right\|\right), \\
& \leq\left[\sum_{i=1}^{n}\left|\mathcal{R}^{k, i}(s)\right|\right]\left\|\dot{\mathbf{U}}^{k}(s)-\dot{\mathbf{U}}^{k}\left(t_{1}\right)\right\|,
\end{aligned}
$$


and integrating over $\left[t_{1}, t\right]$ :

$$
\begin{aligned}
\frac{1}{2}\left\|\dot{\mathbf{U}}^{k}(t)-\dot{\mathbf{U}}^{k}\left(t_{1}\right)\right\|^{2} \leq & \int_{t_{1}}^{t}\left[\sum_{i=1}^{n}\left|\mathcal{R}^{k, i}(s)\right|\right]\left\|\dot{\mathbf{U}}^{k}(s)-\dot{\mathbf{U}}^{k}\left(t_{1}\right)\right\|+\int_{t_{1}}^{t} \mathcal{F}\left(s, \mathbf{U}^{k}(s), \dot{\mathbf{U}}^{k}(s)\right) \cdot\left(\dot{\mathbf{U}}^{k}(s)-\dot{\mathbf{U}}^{k}\left(t_{1}\right)\right), \\
\leq & \int_{t_{1}}^{t}\left[\left\|\mathcal{F}\left(s, \mathbf{U}_{0}, \mathbf{V}_{0}\right)\right\|+\sum_{i=1}^{n}\left|\mathcal{R}^{k, i}(s)\right|\right]\left\|\dot{\mathbf{U}}^{k}(s)-\dot{\mathbf{U}}^{k}\left(t_{1}\right)\right\| \\
& +\kappa \int_{t_{1}}^{t}\left(\left\|\mathbf{U}^{k}(s)-\mathbf{U}_{0}\right\|+\left\|\dot{\mathbf{U}}^{k}(s)-\mathbf{V}_{0}\right\|\right) \cdot\left\|\dot{\mathbf{U}}^{k}(s)-\dot{\mathbf{U}}^{k}\left(t_{1}\right)\right\| .
\end{aligned}
$$

Again using Lemma A.5, p. 157 of [4], we obtain the estimate:

$$
\left\|\dot{\mathbf{U}}^{k}\left(t_{2}\right)-\dot{\mathbf{U}}^{k}\left(t_{1}\right)\right\| \leq \int_{t_{1}}^{t_{2}}\left\|\mathcal{F}\left(s, \mathbf{U}_{0}, \mathbf{V}_{0}\right)\right\|+\sum_{i=1}^{n}\left|\mathcal{R}^{k, i}(s)\right|+\kappa\left\|\mathbf{U}^{k}(s)-\mathbf{U}_{0}\right\|+\kappa\left\|\dot{\mathbf{U}}^{k}(s)-\mathbf{V}_{0}\right\| .
$$

Taking the limit $k \rightarrow \infty$, we finally obtain:

$$
\left\|\dot{\mathbf{U}}\left(t_{2}\right)-\dot{\mathbf{U}}\left(t_{1}\right)\right\| \leq \int_{t_{1}}^{t_{2}}\left\|\mathcal{F}\left(s, \mathbf{U}_{0}, \mathbf{V}_{0}\right)\right\|+\sum_{i=1}^{n}\left|\mathcal{R}^{i}(s)\right|+2 \kappa\|\mathbf{U}\|_{C^{0}}+2 \kappa\|\dot{\mathbf{U}}\|_{C^{0}} .
$$

which shows that the function $\dot{\mathbf{U}}$ is absolutely continuous: $\dot{\mathbf{U}} \in W^{1,1}\left(0, T ; \mathbb{R}^{\text {nd }}\right)$. Furthermore, going back to estimate (1), we can write:

$$
\left\|\ddot{\mathbf{U}}^{k}(t)\right\| \leq\left\|\mathcal{F}\left(t, \mathbf{U}_{0}, \mathbf{V}_{0}\right)\right\|+\sum_{i=1}^{n}\left|\mathcal{R}^{k, i}(t)\right|+\kappa\left\|\mathbf{U}^{k}(t)-\mathbf{U}_{0}\right\|+\kappa\left\|\dot{\mathbf{U}}^{k}(t)-\mathbf{V}_{0}\right\|,
$$

for almost all $t \in[0, T]$ and all $k \in \mathbb{N}$. Therefore:

$$
\left\|\ddot{\mathbf{U}}^{k}\right\|_{L^{1}} \leq M
$$

for some constant independent of $k$. Therefore, extracting a sub-sequence if necessary, the sequence $\left(\ddot{\mathbf{U}}^{k}\right)$ converges in $\mathcal{M}\left([0, T] ; \mathbb{R}^{n d}\right)$ weak-*. Its limit is necessarily $\ddot{\mathbf{U}}$. Furthermore, the differential inclusion under consideration can be given the following equivalent form.

$\forall i \in\{1, \ldots, n\}, \quad \forall \mathbf{v} \in C^{0}\left([0, T] ; \mathbb{R}^{d-1}\right)$,

$$
\int_{0}^{T}\left|\mathcal{R}^{k, i}\right| \cdot\|\mathbf{v}\| \geq \int_{0}^{T}\left|\mathcal{R}^{k, i}\right| \cdot\left\|\dot{\mathbf{u}}_{\mathrm{t}}^{k, i}\right\|+\int_{0}^{T}\left[\ddot{\mathbf{u}}_{\mathrm{t}}^{k, i}-\mathcal{F}^{i}\left(s, \mathbf{U}^{k}, \dot{\mathbf{U}}^{k}\right)\right] \cdot\left(\mathbf{v}+\dot{\mathbf{u}}_{\mathrm{t}}^{k, i}\right) .
$$

Thanks to the convergence properties of all the sequences involved, we can take the limit as $k \rightarrow \infty$ in this inequality. We deduce that $\mathbf{U}$ is a solution of the evolution problem under consideration.

Step 3. Proof of Proposition 3.1 in the general case.

Let $\left(\mathbf{U}^{k}\right)$ be the sequence in $W^{2,1}\left(0, T ; \mathbb{R}^{n d}\right)$ defined by its first term:

$$
\mathbf{U}=\mathbf{U}_{0}+t \mathbf{V}_{0},
$$

and the following induction: given $\mathbf{U}^{k}$, the function $\mathbf{U}^{k+1}$ is defined as the unique solution, given by step 2 , of the following evolution problem.

- $\mathbf{U}^{k+1}(0)=\mathbf{U}_{0} \quad ; \quad \dot{\mathbf{U}}^{k+1}(0)=\mathbf{V}_{0}$ (initial condition),

- $\ddot{\mathbf{U}}^{k+1}(t)-\mathcal{F}\left(t, \mathbf{U}^{k+1}(t), \dot{\mathbf{U}}^{k+1}(t)\right) \in \partial S_{\left|\mathcal{R}\left(t, \mathbf{U}^{k}(t), \dot{\mathbf{U}}^{k}(t)\right)\right| \cdot \bar{C}}\left[-\dot{\mathbf{U}}^{k+1}(t)\right]$, for a.a. $t$. 
Using the same algebra as at the beginning of step 2 and applying Lemma A.5, p 157 of [4] gives:

$$
\begin{aligned}
\left\|\dot{\mathbf{U}}^{k+1}(t)-\dot{\mathbf{U}}^{k}(t)\right\| & \leq \int_{0}^{t}\left\|\mathcal{F}\left(s, \mathbf{U}^{k+1}, \dot{\mathbf{U}}^{k+1}\right)-\mathcal{F}\left(s, \mathbf{U}^{k}, \dot{\mathbf{U}}^{k}\right)\right\|+\int_{0}^{t}\left|\mathcal{R}\left(s, \mathbf{U}^{k}, \dot{\mathbf{U}}^{k}\right)-\mathcal{R}\left(s, \mathbf{U}^{k-1}, \dot{\mathbf{U}}^{k-1}\right)\right|, \\
& \leq \kappa(1+T) \int_{0}^{t}\left\|\dot{\mathbf{U}}^{k+1}-\dot{\mathbf{U}}^{k}\right\|+\kappa^{\prime}(1+T) \int_{0}^{t}\left\|\dot{\mathbf{U}}^{k}-\dot{\mathbf{U}}^{k-1}\right\|,
\end{aligned}
$$

where $\kappa^{\prime}=\kappa \max _{i} \mu_{i}$ is the Lipschitz modulus of the function $\mathcal{R}$. Using the Gronwall-Bellman lemma (Lem. A.4, p. 156 of $[4])$ :

$$
\begin{aligned}
\forall t \in[0, T], \quad\left\|\dot{\mathbf{U}}^{k+1}(t)-\dot{\mathbf{U}}^{k}(t)\right\| & \leq \mathrm{e}^{\kappa T(1+T)} \kappa^{\prime}(1+T) \int_{0}^{t}\left\|\dot{\mathbf{U}}^{k}-\dot{\mathbf{U}}^{k-1}\right\|, \\
& \leq \frac{\left[\kappa^{\prime} t(1+T) \mathrm{e}^{\kappa T(1+T)}\right]^{k}}{k !}\left\|\dot{\mathbf{U}}^{1}-\dot{\mathbf{U}}^{0}\right\|_{C^{0}},
\end{aligned}
$$

which shows that since $\left(\dot{\mathbf{U}}^{k}\right)$ is a Cauchy sequence in the Banach space $C^{0}\left([0, T] ; \mathbb{R}^{\text {nd }}\right)$, it converges in that space towards a limit denoted by $\mathbf{V}$. Setting $\mathbf{U} \stackrel{\text { def }}{=} \mathbf{U}_{0}+\int_{0}^{t} \mathbf{V}$, it can be readily checked that $\left(\mathbf{U}^{k}\right)$ converges towards $\mathbf{U}$ in $C^{0}$ and that $\dot{\mathbf{U}}=\mathbf{V}$. Based on exactly the same reasoning as at the end of step 1, we deduce that $\mathbf{U}$ solves the evolution problem under consideration and that it is the only solution.

\subsection{The bilateral problem with analytic force}

The aim of this section is to prove that if the functions $\mathcal{F}(t, \mathbf{U}, \mathbf{V})$ and $\mathcal{R}(t, \mathbf{U}, \mathbf{V})$ are assumed in addition to be analytic, then the solution of problem $\mathcal{P}_{\mathbf{b}}$ given by Proposition 3.1, is also analytic on a right-neighborhood of $t=0$.

From now on, we assume that the function $\mathbf{F}:[0, T] \times \mathbb{R}^{n d} \times \mathbb{R}^{n d} \rightarrow \mathbb{R}^{\text {nd }}$ satisfies the following hypotheses:

(i) the function $(\mathbf{U}, \mathbf{V}) \mapsto \mathbf{F}(t, \mathbf{U}, \mathbf{V})$ satisfies a Lipschitz condition of modulus $\kappa \geq 0$, for all $t \in[0, T]$ :

$$
\begin{aligned}
\forall t \in[0, T], \quad \forall\left(\mathbf{U}_{1}, \mathbf{V}_{1}\right),\left(\mathbf{U}_{2}, \mathbf{V}_{2}\right) \in \mathbb{R}^{n d} \times \mathbb{R}^{n d}, \\
\\
\left\|\mathbf{F}\left(t, \mathbf{U}_{1}, \mathbf{V}_{1}\right)-\mathbf{F}\left(t, \mathbf{U}_{2}, \mathbf{V}_{2}\right)\right\| \leq \kappa\left\|\left(\mathbf{U}_{1}, \mathbf{V}_{1}\right)-\mathbf{F}\left(t, \mathbf{U}_{2}, \mathbf{V}_{2}\right)\right\| .
\end{aligned}
$$

(ii) the function $(t, \mathbf{U}, \mathbf{V}) \mapsto \mathbf{F}(t, \mathbf{U}, \mathbf{V})$ is analytic,

These hypotheses are also satisfied by the two functions $\mathcal{F}(t, \mathbf{U}, \mathbf{V})$ and $\mathcal{R}(t, \mathbf{U}, \mathbf{V})$. With this additional hypothesis, the solution of problem $\mathcal{P}_{\mathbf{b}}$ fulfils the following regularity result.

Proposition 3.2. There exists $\eta>0$ such that the restriction to $\left[0, \eta\left[\right.\right.$ of the solution $\mathbf{U} \in W^{2,1}\left([0, T] ; \mathbb{R}^{\text {nd }}\right)$ to problem $\mathcal{P}_{\mathbf{b}}$, resulting from Proposition 3.1, is analytic.

Proof. The strategy on which the proof will be based is as follows. We will look for a solution as a formal power series $\mathbf{S}=\sum_{k=0}^{\infty} \mathfrak{U}_{k} t^{k}$ and will attempt to identify one term after another. Since the zeroes of an analytic function of time cannot accumulate, the same is expected to be true of the switches between the stick and slip status of each particle in the system. Therefore, the analysis will consist in determining the status of each particle (stick or slip) in a right-neighborhood of the time origin. The status of each particle is naturally given by the first non-zero term in the formal power expansion of its displacement, but higher order analysis is necessary because of the couplings between particles. Once the status of each particle (stick or slip) has been determined, the evolution problem can be written in the form of an ordinary differential equation, and the analyticity of its solution is given by classical theorems. To conclude, it will then suffice to prove that a restriction of this analytic function to a right-neighborhood of the time origin yields a restriction of the unique solution to problem $\mathcal{P}_{\mathbf{b}}$. 
Step 1. Calculating the $\mathfrak{U}_{k}$.

Initializing. The first two terms $\mathfrak{U}_{0}$ and $\mathfrak{U}_{1}$ in the formal power series $\mathbf{S}=\sum_{k=0}^{\infty} \mathfrak{U}_{k} t^{k}$ are obviously given by the initial condition:

$$
\mathfrak{U}_{0}=\mathbf{U}_{0}, \quad \mathfrak{U}_{1}=\mathbf{V}_{0} .
$$

The calculation of $\mathfrak{U}_{2}$ runs as follows. The vector $\mathfrak{U}_{2} \in \mathbb{R}^{\text {nd }}$ is obtained from the contributions $\mathfrak{u}_{2}^{i} \in \mathbb{R}^{d}$ $(i \in\{1, \ldots, n\})$ of each particle, so that identifying $\mathfrak{U}_{2}$ is equivalent to determining each of the $\mathfrak{u}_{2}^{i}$.

- With $i \in \mathcal{I}$, the corresponding particles are not in active contact with an obstacle so that their reaction force vanishes and the equation of motion gives:

$$
\forall i \in \mathcal{I}, \quad 2 \mathfrak{u}_{2}^{i}=\mathcal{F}^{i}\left(0, \mathbf{U}_{0}, \mathbf{V}_{0}\right)=\mathbf{f}^{i}\left(0, \mathbf{U}_{0}, \mathbf{V}_{0}\right) .
$$

- With $i \in \mathcal{G}_{0} \stackrel{\text { def }}{=}\left\{i \in \mathcal{B} \mid \mathbf{v}_{0}^{i} \neq \mathbf{0}\right\}$, the corresponding particles are slipping in a right-neighborhood of the time origin. The $\mathfrak{u}_{2}^{i}$ are given by:

$$
\forall i \in \mathcal{G}_{0}, \quad 2 \mathfrak{u}_{2}^{i}=\mathcal{F}^{i}\left(0, \mathbf{U}_{0}, \mathbf{V}_{0}\right)-\left|\mathcal{R}^{i}\left(0, \mathbf{U}_{0}, \mathbf{V}_{0}\right)\right| \frac{\mathbf{v}_{0}^{i}}{\left\|\mathbf{v}_{0}^{i}\right\|}
$$

- With $i \in \mathcal{A}_{0} \stackrel{\text { def }}{=}\left\{i \in \mathcal{B} \mid \mathbf{v}_{0}^{i}=\mathbf{0}\right.$ and $\left.\left\|\mathcal{F}^{i}\left(0, \mathbf{U}_{0}, \mathbf{V}_{0}\right)\right\|<\left|\mathcal{R}^{i}\left(0, \mathbf{U}_{0}, \mathbf{V}_{0}\right)\right|\right\}$, the corresponding particles are sticking in a right-neighborhood of the time origin and:

$$
\forall i \in \mathcal{A}_{0}, \quad \mathfrak{u}_{2}^{i}=\mathbf{0}=\mathfrak{u}_{k}^{i} \quad(k \in \mathbb{N}) .
$$

- With $i \in \mathcal{B} \backslash\left(\mathcal{G}_{0} \cup \mathcal{A}_{0}\right)$, the particle has vanishing velocity and its associated reaction force is exactly at the friction threshold. Upon examining the motion equation, it can be readily seen that:

$$
\forall i \in \mathcal{B} \backslash\left(\mathcal{G}_{0} \cup \mathcal{A}_{0}\right), \quad \mathfrak{u}_{2}^{i}=\mathbf{0},
$$

and the status of these particles (stick or slip) must be determined by higher order analysis.

Induction. We assume that the expected formal power expansion has been calculated up to order $p+2$ :

$$
\mathbf{S}_{p+2}=\sum_{k=0}^{p+2} \mathfrak{U}_{k} t^{k},
$$

and that it is associated with two non-intersecting subsets $\mathcal{G}_{p}$ and $\mathcal{A}_{p}$ of $\mathcal{B}$. These particles in $\mathcal{G}_{p}$ are those particles that have been determined as being slipping particles because the formal power series expansion of their displacement has a non-zero term of an order no larger than $p+2$. These particles in $\mathcal{A}_{p}$ are those that have been determined as being sticking particles because the reaction force associated with the partial (up to order $p$ ) formal power series expansion of their displacement is not at the friction threshold. The particles in $\mathcal{B} \backslash\left(\mathcal{G}_{p} \cup \mathcal{A}_{p}\right)$ are those whose status (stick or slip) is still undetermined at order $p+2$, because they have a vanishing velocity at order $p+2$ and the corresponding reaction force at order $p$ is at the friction threshold.

Given a formal power series, we introduce the following notation for extracting the coefficient of order $p$ :

$$
\left[\sum_{k=0}^{\infty} \mathfrak{a}_{k} t^{k}\right]_{p}=\mathfrak{a}_{p} .
$$

The calculation of $\mathfrak{U}_{p+3}$ runs as follows.

- With $i \in \mathcal{I}$, the corresponding particles are not in active contact with an obstacle so that their reaction force vanishes and the equation of motion gives:

$$
\forall i \in \mathcal{I}, \quad(p+2)(p+3) \mathfrak{u}_{p+3}^{i}=\left[\mathcal{F}^{i}\left(t, \mathbf{S}_{p+2}(t), \dot{\mathbf{S}}_{p+2}(t)\right)\right]_{p+1}=\left[\mathbf{f}^{i}\left(t, \mathbf{S}_{p+2}(t), \dot{\mathbf{S}}_{p+2}(t)\right)\right]_{p+1} .
$$


- With $i \in \mathcal{G}_{p+1} \stackrel{\text { def }}{=} \mathcal{G}_{p} \cup\left\{i \in \mathcal{B} \backslash\left(\mathcal{G}_{p} \cup \mathcal{A}_{p}\right)\left|\left\|\left[\mathcal{F}^{i}\left(t, \mathbf{S}_{p+2}, \dot{\mathbf{S}}_{p+2}\right)\right]_{p+1}\right\|>\right|\left[\mathcal{R}^{i}\left(t, \mathbf{S}_{p+2}, \dot{\mathbf{S}}_{p+2}\right)\right]_{p+1} \mid\right\}$, the corresponding particles are slipping and $\mathfrak{u}_{p+3}^{i}$ is the unique solution of:

$$
(p+2)(p+3) \mathfrak{u}_{p+3}^{i}=\left[\mathcal{F}^{i}\left(t, \mathbf{S}_{p+2}(t), \dot{\mathbf{S}}_{p+2}(t)\right)\right]_{p+1}-\left|\left[\mathcal{R}^{i}\left(t, \mathbf{S}_{p+2}(t), \dot{\mathbf{S}}_{p+2}(t)\right)\right]_{p+1}\right| \frac{\sum_{k=1}^{p+3} k \mathfrak{u}_{k}^{i} t^{k-1}}{\left\|\sum_{k=1}^{p+3} k \mathfrak{u}_{k}^{i} t^{k-1}\right\|}
$$

- With $i \in \mathcal{A}_{p+1} \stackrel{\text { def }}{=} \mathcal{A}_{p} \cup\left\{i \in \mathcal{B} \backslash\left(\mathcal{G}_{p} \cup \mathcal{A}_{p}\right)\left|\left\|\left[\mathcal{F}^{i}\left(t, \mathbf{S}_{p+2}, \dot{\mathbf{S}}_{p+2}\right)\right]_{p+1}\right\|<\right|\left[\mathcal{R}^{i}\left(t, \mathbf{S}_{p+2}, \dot{\mathbf{S}}_{p+2}\right)\right]_{p+1} \mid\right\}$, the corresponding particles are sticking and $\mathfrak{u}_{p+3}^{i}=\mathbf{0}$.

- With $i \in \mathcal{B} \backslash\left(\mathcal{G}_{p+1} \cup \mathcal{A}_{p+1}\right)$, the status of the corresponding particles is still undetermined at this order and $\mathfrak{u}_{p+3}^{i}=\mathbf{0}$.

Step 2. Construction of the analytic solution from the formal series expansion.

The formal series expansion $\mathbf{S}=\sum_{k=0}^{\infty} \mathfrak{U}_{k} t^{k}$ is now determined from step 1, and we have to ensure that the convergence radius is non-zero. First, note that the two sequences of sets $\left(\mathcal{G}_{p}\right)$ and $\left(\mathcal{A}_{p}\right)$ are non-decreasing. Therefore, they must be constant when $p$ is larger than a certain integer $p_{0}$. We define a remainder $\tilde{\mathbf{U}}(t)$ by:

$$
\widetilde{\mathbf{U}}(t)=\frac{\mathbf{U}(t)-\mathbf{S}_{p_{0}+2}(t)}{t^{p_{0}+2}},
$$

so that $\widetilde{\mathbf{U}}(0)=\mathbf{0}$. Here it is proposed to use the known status of all the particles at order $p=p_{0}$ to put the evolution problem in the form of an ordinary differential equation. If $i \in \mathcal{G}_{p_{0}}$ then we set $\operatorname{sign}^{i}=+1$ or -1 depending on the sign of the first non-zero term in the formal series $\mathcal{R}^{i}(t, \mathbf{S}(t), \dot{\mathbf{S}}(t))$. If it is identically vanishing, then we set $\operatorname{sign}^{i}=+1$ (this arbitrary choice will be of no consequence). We then take:

$$
\begin{aligned}
& \tilde{\mathbf{U}}(t)=\frac{\mathbf{U}(t)-\mathbf{S}_{p_{0}+2}(t)}{t^{p_{0}+2}}, \\
& \tilde{\mathbf{V}}(t)=\frac{\dot{\mathbf{U}}(t)-\dot{\mathbf{S}}_{p_{0}+2}(t)}{t^{p_{0}+1}},
\end{aligned}
$$

so that, $(\widetilde{\mathbf{U}}(t), \widetilde{\mathbf{V}}(t))$ must satisfy the following Cauchy problem.

Problem $\mathcal{C}$. Find two functions $(\widetilde{\mathbf{U}}(t), \widetilde{\mathbf{V}}(t)$ such that:

- $\tilde{\mathbf{U}}(0)=\mathbf{0} \quad ; \quad \widetilde{\mathbf{V}}(0)=\mathbf{0}$

- $\frac{\mathrm{d} \widetilde{\mathbf{U}}(t)}{\mathrm{d} t}=\frac{\tilde{\mathbf{V}}(t)-\left(p_{0}+2\right) \widetilde{\mathbf{U}}(t)}{t} ;$

- $\forall i \in \mathcal{B} \backslash \mathcal{G}_{p_{0}}, \quad \widetilde{\mathbf{u}}^{i} \equiv \mathbf{0}, \quad \widetilde{\mathbf{v}}^{i} \equiv \mathbf{0}$;

- $\forall i \in \mathcal{I} \cup \mathcal{G}_{p_{0}}, \quad \frac{\mathrm{d} \widetilde{\mathbf{v}}^{i}(t)}{\mathrm{d} t}=-\frac{\left(p_{0}+1\right) \widetilde{\mathbf{v}}^{i}}{t}+\widetilde{\mathbf{g}}^{i}(t, \widetilde{\mathbf{U}}(t), \tilde{\mathbf{V}}(t))$;

where we have taken:

$$
\begin{aligned}
\widetilde{\mathcal{F}}(t, \widetilde{\mathbf{U}}, \widetilde{\mathbf{V}}) & =\mathcal{F}\left(t, \mathbf{S}_{p_{0}+2}(t)+t^{p_{0}+2} \widetilde{\mathbf{U}}, \dot{\mathbf{S}}_{p_{0}+2}(t)+t^{p_{0}+1} \widetilde{\mathbf{V}}\right), \\
\widetilde{\mathcal{R}}(t, \widetilde{\mathbf{U}}, \widetilde{\mathbf{V}}) & =\mathcal{R}\left(t, \mathbf{S}_{p_{0}+2}(t)+t^{p_{0}+2} \widetilde{\mathbf{U}}, \dot{\mathbf{S}}_{p_{0}+2}(t)+t^{p_{0}+1} \widetilde{\mathbf{V}}\right), \\
\widetilde{\mathbf{g}}^{i}(t, \widetilde{\mathbf{U}}, \widetilde{\mathbf{V}}) & =\frac{1}{t^{p_{0}+1}}\left[\widetilde{\mathcal{F}}^{i}(t, \widetilde{\mathbf{U}}, \widetilde{\mathbf{V}})-\ddot{\mathbf{S}}_{p_{0}+2}(t)-\operatorname{sign}^{i} \widetilde{\mathcal{R}}^{i}(t, \widetilde{\mathbf{U}}, \widetilde{\mathbf{V}}) \frac{\dot{\mathbf{S}}_{p_{0}+2}^{i}(t)+t^{p_{0}+1} \widetilde{\mathbf{v}}^{i}}{\left\|\dot{\mathbf{S}}_{p_{0}+2}^{i}(t)+t^{p_{0}+1} \widetilde{\mathbf{v}}^{i}\right\|}\right] .
\end{aligned}
$$


It should be clear that the function $\widetilde{\mathcal{F}}$ and $\widetilde{\mathcal{R}}$ are analytic in a neighborhood of $(0, \mathbf{0}, \mathbf{0})$. This is also true of the functions $\widetilde{\mathbf{g}}^{i}\left(i \in \mathcal{G}_{p_{0}}\right)$ since based on the construction of $p_{0}, \dot{\mathbf{S}}_{p_{0}+2}^{i}$ has at least one non-zero term, and the power series expansion of the term between brackets does not show any $t^{p}$ with $p \leq p_{0}$. Thanks to this analyticity, problem $\mathcal{C}$ has a local analytic function $(\widetilde{\mathbf{U}}(t), \widetilde{\mathbf{V}}(t))$ by virtue of Lemma 3.4 of [3]. This solution is unique in the sense that any other solution is either a restriction or an analytic extension of this local solution, which we will assume to be defined in $[0, \eta[(\eta>0)$. We set:

$$
\forall t \in\left[0, \eta\left[, \quad \mathbf{U}(t)=\mathbf{S}_{p_{0}+2}(t)+t^{p_{0}+2} \widetilde{\mathbf{U}}(t) .\right.\right.
$$

By rewinding the algebra of the construction of the formal series $\mathbf{S}$, the following statements can then be readily checked.

- If $i \in \mathcal{I}$, then:

$$
\forall t \in\left[0, \eta\left[, \quad \ddot{\mathbf{u}}^{i}(t)=\mathcal{F}^{i}(t, \mathbf{U}(t), \dot{\mathbf{U}}(t)) .\right.\right.
$$

- If $i \in \mathcal{G}_{p_{0}}$, then:

$$
\forall t \in\left[0, \eta\left[, \quad \ddot{\mathbf{u}}^{i}(t)=\mathcal{F}^{i}(t, \mathbf{U}(t), \dot{\mathbf{U}}(t))-\left|\mathcal{R}^{i}(t, \mathbf{U}(t), \dot{\mathbf{U}}(t))\right| \frac{\dot{\mathbf{u}}^{i}(t)}{\left\|\dot{\mathbf{u}}^{i}(t)\right\|} .\right.\right.
$$

- If $i \in \mathcal{A}_{p_{0}}$, then the leading term of the formal series $\mathcal{R}^{i}(t, \mathbf{U}(t), \dot{\mathbf{U}}(t))-\mathcal{R}^{i}\left(0, \mathbf{U}_{0}, \mathbf{V}_{0}\right)$ is of degree $p_{1} \leq p_{0}$, based on the construction of $\mathcal{A}_{p_{0}}$, and:

$$
\forall t \in] 0, \eta\left[, \quad\left\|\mathcal{F}^{i}\left(t, \mathbf{S}_{p_{1}+2}(t), \dot{\mathbf{S}}_{p_{1}+2}(t)\right)\right\|<\left|\mathcal{R}^{i}\left(t, \mathbf{S}_{p_{1}+2}(t), \dot{\mathbf{S}}_{p_{1}+2}(t)\right)\right|,\right.
$$

after possibly taking a smaller $\eta$ if necessary. This ensures that the tangential reaction force of that particle does not reach the friction threshold in the time interval $] 0, \eta[$.

- If $i \in \mathcal{B} \backslash\left(\mathcal{G}_{p_{0}} \cup \mathcal{A}_{p_{0}}\right)$, the corresponding particle remains at rest. We now have to prove that the tangential reaction force remains at the friction threshold. If this was not true, based on the analyticity of $\mathcal{F}^{i}$ and $\mathcal{R}^{i}$, we would have:

$$
\begin{aligned}
& \mathcal{F}^{i}(t, \mathbf{U}(t), \dot{\mathbf{U}}(t))-\mathcal{F}^{i}\left(0, \mathbf{U}_{0}, \mathbf{V}_{0}\right) \sim \alpha t^{r}, \\
& \mathcal{R}^{i}(t, \mathbf{U}(t), \dot{\mathbf{U}}(t))-\mathcal{R}^{i}\left(0, \mathbf{U}_{0}, \mathbf{V}_{0}\right) \sim \beta t^{s},
\end{aligned}
$$

with some $r, s \in \mathbb{N}$ and $\alpha \in \mathbb{R}^{d-1}, \beta \in \mathbb{R}$ such that $r \neq s$ or $\|\alpha\| \neq|\beta|$. Setting $p_{1}=\min (r, s)-2$ and noting that $\mathcal{F}^{i}(t, \mathbf{U}(t), \dot{\mathbf{U}}(t))$ and $\mathcal{F}^{i}\left(t, \mathbf{S}_{p_{1}+2}(t), \dot{\mathbf{S}}_{p_{1}+2}(t)\right)$ have the same power expansion at order $p_{1}+2$, one could conclude that either $i \in \mathcal{G}_{p_{1}}$, or $i \in \mathcal{A}_{p_{1}}$ which constitutes a contradiction.

Therefore, restricting the analytic function $\mathbf{U}(t)$ to a right-neighborhood of the time origin solves problem $\mathcal{P}_{\mathbf{b}}$.

\section{The Unilateral PROBLEM With ANALYTIC FORCE}

\subsection{Existence of a local analytic solution}

Proposition 4.1. Let $\mathbf{F}:[0, T] \times \mathbb{R}^{\text {nd }} \times \mathbb{R}^{\text {nd }} \rightarrow \mathbb{R}^{\text {nd }}$ be a given function satisfying hypotheses (i) and (ii) in Theorem 2.1. Then, there exist $\left.\left.T_{\mathrm{a}} \in\right] 0, T\right]$ and some analytic functions $\mathbf{U}_{\mathrm{a}}:\left[0, T_{\mathrm{a}}\right] \rightarrow \mathbb{R}^{n d}, \mathbf{R}_{\mathrm{a}, \mathrm{n}}:\left[0, T_{\mathrm{a}}\right] \rightarrow \mathbb{R}^{n}$ such that:

- $\mathbf{U}_{\mathrm{a}}(0)=\mathbf{U}_{0}, \quad \dot{\mathbf{U}}_{\mathrm{a}}(0)=\mathbf{V}_{0}$;

- $\ddot{\mathbf{U}}_{\mathrm{a}, \mathrm{n}}(t)=\mathbf{F}_{\mathrm{n}}\left(t, \mathbf{U}_{\mathrm{a}}(t), \dot{\mathbf{U}}_{\mathrm{a}}(t)\right)+\mathbf{R}_{\mathrm{a}, \mathrm{n}}(t), \quad \forall t \in\left[0, T_{\mathrm{a}}\right]$;

- $\ddot{\mathbf{U}}_{\mathrm{a}, \mathrm{t}}(t)-\mathbf{F}_{\mathrm{t}}\left(t, \mathbf{U}_{\mathrm{a}}(t), \dot{\mathbf{U}}_{\mathrm{a}}(t)\right) \in \partial S_{\left(\mu_{j} r_{\mathrm{a}, \mathrm{n}}^{j}\right) \cdot \bar{B}}\left[-\dot{\mathbf{U}}_{\mathrm{a}, \mathrm{t}}(t)\right], \quad \forall t \in\left[0, T_{\mathrm{a}}\right]$;

- $\mathbf{U}_{\mathrm{a}, \mathrm{n}}(t) \leq 0, \quad \mathbf{R}_{\mathrm{a}, \mathrm{n}}(t) \leq 0, \quad \mathbf{U}_{\mathrm{a}, \mathrm{n}}(t) \cdot \mathbf{R}_{\mathrm{a}, \mathrm{n}}(t)=0, \quad \forall t \in\left[0, T_{\mathrm{a}}\right]$; 
where $\left(\mu_{j} r_{\mathrm{a}, \mathrm{n}}^{j}\right)$ denotes the vector in $\mathbb{R}^{n}$ with components $\mu_{j} r_{\mathrm{a}, \mathrm{n}}^{j}$. In addition any other analytic solution will be either a restriction or an extension of that solution.

Proof. The strategy on which the proof will be based is very similar to that used in the proof of Proposition 3.2. First, we will attempt to identify the solution in the form of formal series $\mathbf{U}=\sum_{k=0}^{\infty} \mathfrak{U}_{k} t^{k}, \mathbf{R}=\sum_{k=0}^{\infty} \mathfrak{R}_{k} t^{k}$ by examining each order $k$ successively. Here, the issue is to determine the contact status (active contact or not) of each particle in a right-neighborhood of the time origin. Once the status (active contact or not) of each particle has been determined (and it will be uniquely determined), Propositions 3.1 and 3.2 will yield analytic functions which coincide with the formal series and give the expected solution.

Step 1. Calculating of the $\mathfrak{U}_{k}$ and $\mathfrak{R}_{k}$.

Initializating. The first two terms $\mathfrak{U}_{0}$ and $\mathfrak{U}_{1}$ in the formal power series $\mathbf{U}=\sum_{k=0}^{\infty} \mathfrak{U}_{k} t^{k}$ are obviously given by the initial condition:

$$
\mathfrak{U}_{0}=\mathbf{U}_{0}, \quad \mathfrak{U}_{1}=\mathbf{V}_{0} .
$$

Set:

$$
\mathcal{I}_{-1} \stackrel{\text { def }}{=}\left\{i \in\{1, \ldots, n\} \mid \mathbf{u}_{0, \mathrm{n}}^{i}<0 \text { or } \mathbf{v}_{0, \mathrm{n}}^{i}<0\right\} \text {. }
$$

The particles with the index $i \in \mathcal{I}_{-1}$ will be in non-active contact and $\mathfrak{r}_{k}^{i}=0$, for all $k \in \mathbb{N}$. For the other particles, the two real numbers $\mathfrak{u}_{2, \mathrm{n}}^{i}$ and $\mathfrak{r}_{0}^{i}$ are uniquely determined by the linear complementarity problem:

- $2 \mathfrak{u}_{2, \mathrm{n}}^{i}=\mathbf{F}_{\mathrm{n}}\left(0, \mathbf{U}_{0}, \mathbf{V}_{0}\right)+\mathfrak{r}_{0}^{i}$

- $\mathfrak{u}_{2, \mathrm{n}}^{i} \leq 0, \quad \mathfrak{r}_{0}^{i} \leq 0, \quad \mathfrak{u}_{2, \mathrm{n}}^{i} \mathfrak{r}_{0}^{i}=0$.

We then set:

$$
\begin{aligned}
& \mathcal{I}_{0}=\mathcal{I}_{-1} \cup\left\{i \in\{1, \ldots, n\} \mid \mathfrak{u}_{2, \mathrm{n}}^{i}<0\right\} \\
& \mathcal{B}_{0}=\left\{i \in\{1, \ldots, n\} \mid \mathfrak{r}_{0}^{i}<0\right\}
\end{aligned}
$$

and examine the following evolution problem:

Problem $\mathcal{C}_{0}$.

$$
\begin{aligned}
& \text { - } \mathbf{U}(0)=\mathbf{U}_{0}, \quad \mathbf{V}(0)=\mathbf{V}_{0} ; \\
& \text { - } \forall i \in \mathcal{B}_{0}, \quad \mid \begin{array}{l}
\ddot{u}_{\mathrm{n}}^{i}=0, \\
\ddot{\mathbf{u}}_{\mathrm{t}}^{i}-\mathbf{f}_{\mathrm{t}}^{i}(t, \mathbf{U}, \dot{\mathbf{U}}) \in \partial S_{\left|\mu_{i} f_{\mathrm{n}}^{i}\right| \cdot \bar{B}}\left[-\dot{\mathbf{u}}_{\mathrm{t}}^{i}\right]
\end{array} \\
& \text { - } \forall i \in\{1, \ldots, n\} \backslash \mathcal{B}_{0}, \quad \ddot{\mathbf{u}}^{i}(t)=\mathbf{f}^{i}(t, \mathbf{U}(t), \dot{\mathbf{U}}(t)) .
\end{aligned}
$$

Problem $\mathcal{C}_{\mathbf{0}}$ has a unique analytic solution by virtue of Proposition 3.2. Let $\mathbf{U}_{2}$ be the power expansion at order 2 of that solution, and let $\mathbf{R}_{0}$ be the power expansion at order 0 of $-f_{\mathrm{n}}^{i}(t, \mathbf{U}(t), \dot{\mathbf{U}}(t))$ for $i \in \mathcal{B}_{0}$, and $\mathbf{0}$ otherwise. It can be readily checked that $\left(\mathbf{U}_{2}, \mathbf{R}_{0}\right)$ is the unique pair of power expansions of orders 2 and 0 , that solves the problem at order 0 .

Induction. Let $p \in \mathbb{N}$. We assume that we have constructed the power expansions $\mathbf{U}_{p+2}, \mathbf{R}_{p}$ associated with the set of indexes $\mathcal{I}_{p}, \mathcal{B}_{p}$, which satisfy the equation of motion at order $p$, which means that:

- if $i \in \mathcal{I}_{p}, \mathfrak{u}_{p+2, \mathrm{n}}^{i}$ has a negative leading term, then $\mathfrak{r}_{p}^{i}$ vanishes and $\mathbf{U}_{p+2}$ ensures that the equation of motion for particle $i$ is fulfilled at order $p$;

- if $i \in \mathcal{B}_{p}, \mathfrak{r}_{p}^{i}$ has a negative leading term, then $\mathfrak{u}_{p+2, \mathrm{n}}^{i}$ vanishes and $\mathbf{U}_{p+2}$ ensures that the equation of motion for particle $i$ is fulfilled at order $p$; 
- if $i \notin \mathcal{I}_{p} \cup \mathcal{B}_{p}$, then $\mathfrak{u}_{p+2, \mathrm{n}}^{i}$ and $\mathfrak{r}_{p}^{i}$ vanish.

With $i \in\{1, \ldots, n\} \backslash\left(\mathcal{I}_{p} \cup \mathcal{B}_{p}\right)$, the two real numbers $\mathfrak{u}_{p+3, \mathrm{n}}^{i}$ and $\mathfrak{r}_{p+1}^{i}$ are uniquely determined by the linear complementarity problem:

$$
\begin{aligned}
& \text { - }(p+3)(p+2) \mathfrak{u}_{p+3, \mathrm{n}}^{i}=\left[f_{\mathrm{n}}^{i}\left(t, \mathbf{U}_{p+2}(t), \dot{\mathbf{U}}_{p+2}(t)\right)\right]_{p+1}+\mathfrak{r}_{p+1}^{i} ; \\
& \text { - } \mathfrak{u}_{p+3, \mathrm{n}}^{i} \leq 0, \quad \mathfrak{r}_{p+1}^{i} \leq 0, \quad \mathfrak{u}_{p+3, \mathrm{n}}^{i} \mathfrak{r}_{p+1}^{i}=0 .
\end{aligned}
$$

This can be used to update the two sets:

$$
\begin{aligned}
& \mathcal{I}_{p+1}=\mathcal{I}_{p} \cup\left\{i \in\{1, \ldots, n\} \backslash\left(\mathcal{I}_{p} \cup \mathcal{B}_{p}\right) \mid \mathfrak{u}_{p+3, \mathrm{n}}^{i}<0\right\}, \\
& \mathcal{B}_{p+1}=\mathcal{B}_{p} \cup\left\{i \in\{1, \ldots, n\} \backslash\left(\mathcal{I}_{p} \cup \mathcal{B}_{p}\right) \mid \mathfrak{r}_{p+3, \mathrm{n}}^{i}<0\right\},
\end{aligned}
$$

and to deal with the following evolution problem.

Problem $\mathcal{C}_{\mathbf{p}+1}$.

$$
\begin{aligned}
& \text { - } \mathbf{U}(0)=\mathbf{U}_{0}, \quad \mathbf{V}(0)=\mathbf{V}_{0} ; \\
& \text { - } \forall i \in \mathcal{B}_{p+1}, \quad \mid \begin{array}{l}
\ddot{u}_{\mathrm{n}}^{i}=0, \\
\ddot{\mathbf{u}}_{\mathrm{t}}^{i}-\mathbf{f}_{\mathrm{t}}^{i}(t, \mathbf{U}, \dot{\mathbf{U}}) \in \partial S_{\left|\mu_{i} f_{\mathrm{n}}^{i}\right| \cdot \bar{B}}\left[-\dot{\mathbf{u}}_{\mathrm{t}}^{i}\right]
\end{array} \\
& \text { - } \quad \forall i \in\{1, \ldots, n\} \backslash \mathcal{B}_{p+1}, \quad \ddot{\mathbf{u}}^{i}(t)=\mathbf{f}^{i}(t, \mathbf{U}(t), \dot{\mathbf{U}}(t)) .
\end{aligned}
$$

Problem $\mathcal{C}_{p+1}$ has a unique analytic solution by virtue of Proposition 3.2. Let $\mathbf{U}_{p+3}$ be the power expansion at order $p+3$ of that solution and $\mathbf{R}_{p+1}$ the power expansion at order $p+1$ of $-f_{\mathrm{n}}^{i}(t, \mathbf{U}(t)$, $\mathbf{U}(t))$ with $i \in \mathbf{B}_{p+1}$ and 0 otherwise. It can be readily checked that $\left(\mathbf{U}_{p+3}, \mathbf{R}_{p+1}\right)$ is the unique pair of power expansions of orders $p+3$ and $p+1$, which solves the problem at order $p+1$.

Step 2. Construction of the analytic solution from the formal series expansion.

The two sequences of sets $\left(\mathcal{I}_{p}\right)$ and $\left(\mathcal{B}_{p}\right)$ are non-decreasing, and must therefore be constant when $p$ is larger than a certain integer $p_{0}$. Denote by $\mathbf{U}_{\mathrm{a}}:\left[0, \eta\left[\rightarrow \mathbb{R}^{\text {nd }}\right.\right.$ the analytic solution of problem $\mathcal{C}_{\mathbf{p}_{\mathbf{0}}}$ and by $\mathbf{R}_{\mathrm{a}, \mathrm{n}}:\left[0, \eta\left[\rightarrow \mathbb{R}^{n}\right.\right.$ the function defined by:

$$
\begin{array}{lll}
\forall i \in \mathcal{B}_{p_{0}}, & r_{\mathrm{a}, \mathrm{n}}^{i}(t)=-f_{\mathrm{n}}^{i}\left(t, \mathbf{U}_{\mathrm{a}}(t), \dot{\mathbf{U}}_{\mathrm{a}}(t)\right), \\
\forall i \in\{1, \ldots, n\} \backslash \mathcal{B}_{p_{0}}, & r_{\mathrm{a}, \mathrm{n}}^{i}(t) & =0 .
\end{array}
$$

The pair $\left(\mathbf{U}_{\mathrm{a}}, \mathbf{R}_{\mathrm{a}, \mathrm{n}}\right)$, which is restricted to an interval $\left[0, \eta^{\prime}\right.$ [ so that all the components of $\mathbf{U}_{\mathrm{a}, \mathrm{n}}(t)$ and $\mathbf{R}_{\mathrm{a}, \mathrm{n}}(t)$ are negative on $] 0, \eta^{\prime}$ [, yields a solution to the bilateral problem $\mathcal{P}_{\mathbf{b}}$, and therefore to the problem under consideration. The uniqueness part is given by the uniqueness of the power series expansion of a solution.

\subsection{Local uniqueness for the unilateral problem with analytic force}

This section gives the proof of the following local uniqueness result in the case of the unilateral problem $\mathcal{P}_{\mathbf{u}}$. At the end of the section, it will be established that Theorem 2.1 is nothing but a corollary to Theorem 4.2.

Theorem 4.2. Let $\mathbf{F}:[0, T] \times \mathbb{R}^{\text {nd }} \times \mathbb{R}^{\text {nd }} \rightarrow \mathbb{R}^{\text {nd }}$ be a given function satisfying hypotheses (i), (ii) and (iii) in Theorem 2.1. Let also $\mathbf{U} \in \operatorname{MMA}\left(\left[0, T^{\prime}\right] ; \mathbb{R}^{n d}\right)$ be an arbitrary solution to problem $\mathcal{P}_{\mathbf{u}}$ in the time interval $\left[0, T^{\prime}\right]$ and let $\mathbf{U}_{\mathrm{a}}:\left[0, T_{\mathrm{a}}\right] \rightarrow \mathbb{R}^{\text {nd }}$ be the analytic solution resulting from Proposition 4.1. Then, the two solutions $\mathbf{U}$ and $\mathbf{U}_{\mathrm{a}}$ are identical in a right-neighborhood $\left[0, \eta\left[\subset\left[0, T^{\prime}\right] \cap\left[0, T_{\mathrm{a}}\right]\right.\right.$ of the time origin. 
Proof. The strategy on which the proof will be based is as follows. As in [2], the aim will be to prove that $r_{\mathrm{a}, \mathrm{n}}^{i} u_{\mathrm{n}}^{i}$ vanishes identically in a right-neighborhood of the time origin, for each $i$ : this result means that each particle has the same status (active or inactive contact) in the two possible solutions $\mathbf{U}$ and $\mathbf{U}_{\mathrm{a}}$. Then, the uniqueness part of Proposition 3.1 shows that $\mathbf{U}$ must be equal to $\mathbf{U}_{\mathrm{a}}$. As in [3], the adaptation of the argument in [2] relies on the fact that the difference between $\mathbf{U}$ and $\mathbf{U}_{\mathrm{a}}$ is controlled by the integral of $\left\|\mathbf{R}_{\mathrm{a}, \mathrm{n}}\right\|$. However, this trick cannot be applied simultaneously to all the particles and we can only apply it, in the first step, to the subset of particles having the largest $r_{\mathrm{a}, \mathrm{n}}$. An induction is then necessary to obtain this result for all the particles.

This proof is also the first occurrence of hypothesis (iii). Let us explain a little bit how it arises. Following the strategy in [3], we will have to estimate:

$$
\int_{[0, t]} u_{\mathrm{n}}^{i} \frac{\mathrm{d}}{\mathrm{d} s}\left[f_{\mathrm{n}}^{i}(s, \mathbf{U}, \dot{\mathbf{U}})-f_{\mathrm{n}}^{i}\left(s, \mathbf{U}_{\mathrm{a}}, \dot{\mathbf{U}}_{\mathrm{a}}\right)\right] .
$$

This is going to be the same problem as estimating $u_{\mathrm{n}}^{i} \ddot{\mathbf{U}}$, that is, $u_{\mathrm{n}}^{i} \mathbf{R}$ for which no estimate is available. However, it is easy to estimate $u_{\mathrm{n}}^{i} \mathbf{r}^{i}$ : it vanishes identically, thanks to the contact condition complemented by the Coulomb law of friction. This means that we are only able to estimate the integral (2) under the supplementary hypothesis (iii), in which case the above integral reduces to:

$$
\int_{[0, t]} u_{\mathrm{n}}^{i} \frac{\mathrm{d}}{\mathrm{d} s}\left[f_{\mathrm{n}}^{i}\left(s, \mathbf{U}, \dot{\mathbf{u}}^{i}\right)-f_{\mathrm{n}}^{i}\left(s, \mathbf{U}_{\mathrm{a}}, \dot{\mathbf{u}}_{\mathrm{a}}^{i}\right)\right] .
$$

Step 1. There exists a real constant $C$ depending only on $\kappa, T$ and $n$ such that:

$$
\forall t \in[0, T], \quad\left\|\dot{\mathbf{U}}^{+}(t)-\dot{\mathbf{U}}_{\mathrm{a}}(t)\right\| \leq C \int_{0}^{t}\left\|\mathbf{R}_{\mathrm{a}, \mathrm{n}}\right\| .
$$

Upon multiplying the normal part of the equation of motion:

$$
\ddot{\mathbf{U}}_{\mathrm{n}}-\ddot{\mathbf{U}}_{\mathrm{a}, \mathrm{n}}=\mathbf{R}_{\mathrm{n}}-\mathbf{R}_{\mathrm{a}, \mathrm{n}}+\mathbf{F}_{\mathrm{n}}(t, \mathbf{U}, \dot{\mathbf{U}})-\mathbf{F}_{\mathrm{n}}\left(t, \mathbf{U}_{\mathrm{a}}, \dot{\mathbf{U}}_{\mathrm{a}}\right),
$$

by $\left(\dot{\mathbf{U}}_{\mathrm{n}}^{+}+\dot{\mathbf{U}}_{\mathrm{n}}^{-}\right) / 2-\dot{\mathbf{U}}_{\mathrm{a}, \mathrm{n}}$ and integrating, we get:

$$
\begin{aligned}
\frac{1}{2}\left\|\dot{\mathbf{U}}_{\mathrm{n}}^{+}(t)-\dot{\mathbf{U}}_{\mathrm{a}, \mathrm{n}}(t)\right\|^{2}=\int_{[0, t]}\left(\mathbf{R}_{\mathrm{n}}-\right. & \left.\mathbf{R}_{\mathrm{a}, \mathrm{n}}\right) \cdot\left(\frac{\dot{\mathbf{U}}_{\mathrm{n}}^{+}+\dot{\mathbf{U}}_{\mathrm{n}}^{-}}{2}-\dot{\mathbf{U}}_{\mathrm{a}, \mathrm{n}}\right) \\
& +\int_{0}^{t}\left(\mathbf{F}_{\mathrm{n}}(s, \mathbf{U}(s), \dot{\mathbf{U}}(s))-\mathbf{F}_{\mathrm{n}}\left(s, \mathbf{U}_{\mathrm{a}}(s), \dot{\mathbf{U}}_{\mathrm{a}}(s)\right)\right) \cdot\left(\dot{\mathbf{U}}_{\mathrm{n}}^{+}(s)-\dot{\mathbf{U}}_{\mathrm{a}, \mathrm{n}}(s)\right) \mathrm{d} s .
\end{aligned}
$$

Noting that:

- $\mathbf{R}_{\mathrm{n}} \cdot\left(\dot{\mathbf{U}}_{\mathrm{n}}^{+}+\dot{\mathbf{U}}_{\mathrm{n}}^{-}\right) \leq 0$ because the impact constitutive law must not increase the kinetic energy during an impact,

- $\dot{\mathbf{U}}_{\mathrm{a}, \mathrm{n}} \leq 0$, by taking a smaller time interval $[0, \eta[$ if necessary,

we obtain:

$$
\frac{1}{2}\left\|\dot{\mathbf{U}}_{\mathrm{n}}^{+}(t)-\dot{\mathbf{U}}_{\mathrm{a}, \mathrm{n}}(t)\right\|^{2} \leq \int_{[0, t]}\left\|\mathbf{R}_{\mathrm{a}, \mathrm{n}}\right\|\left\|\dot{\mathbf{U}}_{\mathrm{n}}^{+}-\dot{\mathbf{U}}_{\mathrm{a}, \mathrm{n}}\right\|+\kappa \int_{0}^{t}\left(\left\|\mathbf{U}-\mathbf{U}_{\mathrm{a}}\right\|+\left\|\dot{\mathbf{U}}^{+}-\dot{\mathbf{U}}_{\mathrm{a}}\right\|\right)\left\|\dot{\mathbf{U}}_{\mathrm{n}}^{+}-\dot{\mathbf{U}}_{\mathrm{a}, \mathrm{n}}\right\|,
$$

which, from Lemma A.5 of [4], gives:

$$
\left\|\dot{\mathbf{U}}_{\mathrm{n}}^{+}(t)-\dot{\mathbf{U}}_{\mathrm{a}, \mathrm{n}}(t)\right\| \leq \int_{[0, t]}\left\|\mathbf{R}_{\mathrm{a}, \mathrm{n}}\right\|+\kappa \int_{0}^{t}\left(\left\|\mathbf{U}-\mathbf{U}_{\mathrm{a}}\right\|+\left\|\dot{\mathbf{U}}^{+}-\dot{\mathbf{U}}_{\mathrm{a}}\right\|\right) .
$$


Likewise multiplying the tangential component of the motion equation by $\dot{\mathbf{U}}_{\mathrm{t}}^{+}-\dot{\mathbf{U}}_{\mathrm{a}, \mathrm{t}}$ and integrating:

$$
\begin{aligned}
\int_{[0, t]}\left(\ddot{\mathbf{U}}_{\mathrm{t}}-\ddot{\mathbf{U}}_{\mathrm{a}, \mathrm{t}}\right) \cdot\left(\dot{\mathbf{U}}_{\mathrm{t}}^{+}-\dot{\mathbf{U}}_{\mathrm{a}, \mathrm{t}}\right)= & \int_{[0, t]}\left(\mathbf{R}_{\mathrm{t}}-\mathbf{R}_{\mathrm{a}, \mathrm{t}}\right) \cdot\left(\dot{\mathbf{U}}_{\mathrm{t}}^{+}-\dot{\mathbf{U}}_{\mathrm{a}, \mathrm{t}}\right) \\
& +\int_{0}^{t}\left(\mathbf{F}_{\mathrm{t}}(s, \mathbf{U}(s), \dot{\mathbf{U}}(s))-\mathbf{F}_{\mathrm{t}}\left(s, \mathbf{U}_{\mathrm{a}}(s), \dot{\mathbf{U}}_{\mathrm{a}}(s)\right)\right) \cdot\left(\dot{\mathbf{U}}_{\mathrm{t}}^{+}(s)-\dot{\mathbf{U}}_{\mathrm{a}, \mathrm{t}}(s)\right) \mathrm{d} s .
\end{aligned}
$$

In addition by using [11], p. 44:

$$
\int_{[0, t]}\left(\ddot{\mathbf{U}}_{\mathrm{t}}-\ddot{\mathbf{U}}_{\mathrm{t}}\right) \cdot\left(\dot{\mathbf{U}}_{\mathrm{t}}^{+}-\dot{\mathbf{U}}_{\mathrm{a}, \mathrm{t}}\right) \geq \frac{1}{2}\left\|\dot{\mathbf{U}}_{\mathrm{t}}^{+}(t)-\dot{\mathbf{U}}_{\mathrm{a}, \mathrm{t}}(t)\right\|^{2}
$$

and the Coulomb friction law:

$$
\begin{aligned}
\int_{[0, t]}\left(\mathbf{R}_{\mathrm{t}}-\mathbf{R}_{\mathrm{a}, \mathrm{t}}\right) \cdot\left(\dot{\mathbf{U}}_{\mathrm{t}}^{+}-\dot{\mathbf{U}}_{\mathrm{a}, \mathrm{t}}\right) & \leq \sum_{i=1}^{n} \mu_{i} \int_{[0, t]}\left(r_{\mathrm{n}}^{i}-r_{\mathrm{a}, \mathrm{n}}^{i}\right) \cdot\left(\left\|\dot{\mathbf{u}}_{\mathrm{t}}^{i+}\right\|-\left\|\dot{\mathbf{u}}_{\mathrm{a}, \mathrm{t}}^{i}\right\|\right), \\
& \leq \sum_{i=1}^{n} \mu_{i} \int_{[0, t]}\left|r_{\mathrm{n}}^{i}-r_{\mathrm{a}, \mathrm{n}}^{i}\right|\left\|\dot{\mathbf{u}}_{\mathrm{t}}^{i+}-\dot{\mathbf{u}}_{\mathrm{a}, \mathrm{t}}^{i}\right\|, \\
& \leq n \bar{\mu} \int_{[0, t]}\left\|\mathbf{R}_{\mathrm{n}}-\mathbf{R}_{\mathrm{a}, \mathrm{n}}\right\|\left\|\dot{\mathbf{U}}_{\mathrm{t}}^{+}-\dot{\mathbf{U}}_{\mathrm{a}, \mathrm{t}}\right\|
\end{aligned}
$$

where $\bar{\mu}=\left(\sum_{i=1}^{n} \mu_{i}\right) / n$. All in all, this gives:

$$
\frac{1}{2}\left\|\dot{\mathbf{U}}_{\mathrm{t}}^{+}(t)-\dot{\mathbf{U}}_{\mathrm{a}, \mathrm{t}}(t)\right\|^{2} \leq n \bar{\mu} \int_{[0, t]}\left\|\mathbf{R}_{\mathrm{n}}-\mathbf{R}_{\mathrm{a}, \mathrm{n}}\right\|\left\|\dot{\mathbf{U}}_{\mathrm{t}}^{+}-\dot{\mathbf{U}}_{\mathrm{a}, \mathrm{t}}\right\|+\kappa \int_{0}^{t}\left(\left\|\mathbf{U}-\mathbf{U}_{\mathrm{a}}\right\|+\left\|\dot{\mathbf{U}}^{+}-\dot{\mathbf{U}}_{\mathrm{a}}\right\|\right)\left\|\dot{\mathbf{U}}_{\mathrm{t}}^{+}-\dot{\mathbf{U}}_{\mathrm{a}, \mathrm{t}}\right\|,
$$

which, from Lemma A.5 of [4], gives:

$$
\left\|\dot{\mathbf{U}}_{\mathrm{t}}^{+}(t)-\dot{\mathbf{U}}_{\mathrm{a}, \mathrm{t}}(t)\right\| \leq n \bar{\mu} \int_{[0, t]}\left\|\mathbf{R}_{\mathrm{n}}-\mathbf{R}_{\mathrm{a}, \mathrm{n}}\right\|+\kappa \int_{0}^{t}\left(\left\|\mathbf{U}-\mathbf{U}_{\mathrm{a}}\right\|+\left\|\dot{\mathbf{U}}^{+}-\dot{\mathbf{U}}_{\mathrm{a}}\right\|\right) .
$$

Upon returning to the normal component of the motion equation, we estimate:

$$
\begin{aligned}
\int_{[0, t]}\left\|\mathbf{R}_{\mathrm{n}}-\mathbf{R}_{\mathrm{a}, \mathrm{n}}\right\| & \leq \int_{0}^{t}\left\|\mathbf{R}_{\mathrm{a}, \mathrm{n}}\right\|+\int_{[0, t]}\left\|\mathbf{R}_{\mathrm{n}}\right\|, \\
& \leq \int_{0}^{t}\left\|\mathbf{R}_{\mathrm{a}, \mathrm{n}}\right\|-\sum_{i=1}^{n} \int_{[0, t]} r_{\mathrm{n}}^{i}, \\
& \leq \int_{0}^{t}\left\|\mathbf{R}_{\mathrm{a}, \mathrm{n}}\right\|-\sum_{i=1}^{n} \int_{[0, t]} r_{\mathrm{a}, \mathrm{n}}^{i}+\ddot{u}_{\mathrm{n}}^{i}-\ddot{u}_{\mathrm{a}, \mathrm{n}}^{i}-f_{\mathrm{n}}^{i}(s, \mathbf{U}(s), \dot{\mathbf{U}}(s))+f_{\mathrm{n}}^{i}\left(s, \mathbf{U}_{\mathrm{a}}(s), \dot{\mathbf{U}}_{\mathrm{a}}(s)\right), \\
& \leq(\sqrt{n}+1) \int_{0}^{t}\left\|\mathbf{R}_{\mathrm{a}, \mathrm{n}}\right\|+\sqrt{n}\left\|\dot{\mathbf{U}}_{\mathrm{n}}^{+}(t)-\dot{\mathbf{U}}_{\mathrm{a}, \mathrm{n}}(t)\right\|+\sqrt{n} \kappa \int_{0}^{t}\left(\left\|\mathbf{U}-\mathbf{U}_{\mathrm{a}}\right\|+\left\|\dot{\mathbf{U}}^{+}-\dot{\mathbf{U}}_{\mathrm{a}}\right\|\right), \\
& \leq(2 \sqrt{n}+1) \int_{0}^{t}\left\|\mathbf{R}_{\mathrm{a}, \mathrm{n}}\right\|+2 \sqrt{n} \kappa \int_{0}^{t}\left(\left\|\mathbf{U}-\mathbf{U}_{\mathrm{a}}\right\|+\left\|\dot{\mathbf{U}}^{+}-\dot{\mathbf{U}}_{\mathrm{a}}\right\|\right),
\end{aligned}
$$

here estimate (3) has been used to obtain the last inequality. It can be used to rewrite inequality (4) as follows:

$$
\left\|\dot{\mathbf{U}}_{\mathrm{t}}^{+}(t)-\dot{\mathbf{U}}_{\mathrm{a}, \mathrm{t}}(t)\right\| \leq n(2 \sqrt{n}+1) \bar{\mu} \int_{0}^{t}\left\|\mathbf{R}_{\mathrm{a}, \mathrm{n}}\right\|+(2 n \sqrt{n} \bar{\mu}+1) \kappa \int_{0}^{t}\left(\left\|\mathbf{U}-\mathbf{U}_{\mathrm{a}}\right\|+\left\|\dot{\mathbf{U}}^{+}-\dot{\mathbf{U}}_{\mathrm{a}}\right\|\right) .
$$


By combining inequalities (3) and (5), we have proved:

$$
\begin{aligned}
\left\|\dot{\mathbf{U}}^{+}(t)-\dot{\mathbf{U}}_{\mathrm{a}}(t)\right\| & \leq\left\|\dot{\mathbf{U}}_{\mathrm{n}}^{+}(t)-\dot{\mathbf{U}}_{\mathrm{a}, \mathrm{n}}(t)\right\|+\left\|\dot{\mathbf{U}}_{\mathrm{t}}^{+}(t)-\dot{\mathbf{U}}_{\mathrm{a}, \mathrm{t}}(t)\right\|, \\
& \leq(1+n(2 \sqrt{n}+1) \bar{\mu}) \int_{0}^{t}\left\|\mathbf{R}_{\mathrm{a}, \mathrm{n}}\right\|+2(n \sqrt{n} \bar{\mu}+1) \kappa(1+T) \int_{0}^{t}\left\|\dot{\mathbf{U}}^{+}-\dot{\mathbf{U}}_{\mathrm{a}}\right\|,
\end{aligned}
$$

which, based on Gronwall's lemma, gives the expected estimate:

$$
\left\|\dot{\mathbf{U}}^{+}(t)-\dot{\mathbf{U}}_{\mathrm{a}}(t)\right\| \leq(1+n(2 \sqrt{n}+1) \bar{\mu}) \mathrm{e}^{2 T(n \sqrt{n} \bar{\mu}+1) \kappa(1+T)} \int_{0}^{t}\left\|\mathbf{R}_{\mathrm{a}, \mathrm{n}}\right\| .
$$

Step 2. Some of the particles are proved to remain in contact with the obstacle in a right-neighborhood of the time origin $t=0$.

If $\mathbf{R}_{\mathrm{a}, \mathrm{n}}$ vanishes identically, then $\mathbf{U} \equiv \mathbf{U}_{\mathrm{a}}$ according to step 1 . We therefore focus on the case where it does not vanish identically. Let $m_{1} \in \mathbb{N}$ be the order of the first non-zero term in its power series expansion at $t=0$. Let $D_{1}$ be the set of indexes of the corresponding particles:

$$
D_{1}=\left\{i \in\{1, \cdots, n\} \mid \exists \alpha_{i} \in \mathbb{R} \backslash\{0\}, \quad r_{\mathrm{a}, \mathrm{n}}^{i}(t) \sim \alpha_{i} t^{m_{1}}\right\} .
$$

Take $i \in D_{1}$ arbitrary. Multiplying the normal component of the equation of motion for particle $i$ by $\left(\dot{u}_{\mathrm{n}}^{i+}+\dot{u}_{\mathrm{n}}^{i-}\right) / 2$ and integrating, we obtain:

$$
\frac{1}{2}\left|\dot{u}_{\mathrm{n}}^{i+}(t)\right|^{2} \leq-\int_{0}^{t} r_{\mathrm{a}, \mathrm{n}}^{i} \dot{u}_{\mathrm{n}}^{i+}+\int_{0}^{t}\left[f_{\mathrm{n}}^{i}\left(s, \mathbf{U}, \dot{\mathbf{u}}^{i}\right)-f_{\mathrm{n}}^{i}\left(s, \mathbf{U}_{\mathrm{a}}, \dot{\mathbf{u}}_{\mathrm{a}}^{i}\right)\right] \dot{u}_{\mathrm{n}}^{i+},
$$

where the notation recalls the restrictive assumption made about $\mathbf{F}: f^{i}$ does not depend on the full velocity $\dot{\mathbf{U}}$ but only on the velocity $\dot{\mathbf{u}}^{i}$. The latter inequality entails, in particular, that the right-handside is non-negative. Recalling that a Lipschitz-continuous function of a function with bounded variation has bounded variation, it is possible to perform an integration by parts, to obtain the estimate:

$$
r_{\mathrm{a}, \mathrm{n}}^{i}(t) u_{\mathrm{n}}^{i}(t)-\left[f_{\mathrm{n}}^{i}\left(t, \mathbf{U}(t), \dot{\mathbf{u}}^{i}(t)\right)-f_{\mathrm{n}}^{i}\left(t, \mathbf{U}_{\mathrm{a}}(t), \dot{\mathbf{u}}_{\mathrm{a}}^{i}(t)\right)\right] u_{\mathrm{n}}^{i}(t) \leq \int_{0}^{t} \dot{r}_{\mathrm{a}, \mathrm{n}}^{i} u_{\mathrm{n}}^{i}-\int_{[0, t]} u_{\mathrm{n}}^{i} \frac{\mathrm{d}}{\mathrm{d} s}\left[f_{\mathrm{n}}^{i}\left(s, \mathbf{U}, \dot{\mathbf{u}}^{i}\right)-f_{\mathrm{n}}^{i}\left(s, \mathbf{U}_{\mathrm{a}}, \dot{\mathbf{u}}_{\mathrm{a}}^{i}\right)\right],
$$

where the derivative in the last integral is to be understood in the sense of distributions and is nothing but the Stieljes measure of the function with bounded variation to which it applies. No derivative chain rule formula is generally valid in such a situation (see the appendix), but thanks to Proposition A.2 in the appendix, the following inequality between modulus measures holds true:

$$
\begin{aligned}
\mid \frac{\mathrm{d}}{\mathrm{d} s}[ & \left.f_{\mathrm{n}}^{i}\left(s, \mathbf{U}, \dot{\mathbf{u}}^{i}\right)-f_{\mathrm{n}}^{i}\left(s, \mathbf{U}_{\mathrm{a}}, \dot{\mathbf{u}}_{\mathrm{a}}^{i}\right)\right] \mid \\
\leq & \left|\frac{\partial}{\partial s} f_{\mathrm{n}}^{i}\left(s, \mathbf{U}, \dot{\mathbf{u}}^{i}\right)-\frac{\partial}{\partial s} f_{\mathrm{n}}^{i}\left(s, \mathbf{U}_{\mathrm{a}}, \dot{\mathbf{u}}_{\mathrm{a}}^{i}\right)+\dot{\mathbf{U}}^{+} \cdot \frac{\partial}{\partial \mathbf{U}} f_{\mathrm{n}}^{i}\left(s, \mathbf{U}, \dot{\mathbf{u}}^{i}\right)-\dot{\mathbf{U}}_{\mathrm{a}} \cdot \frac{\partial}{\partial \mathbf{U}_{\mathrm{a}}} f_{\mathrm{n}}^{i}\left(s, \mathbf{U}_{\mathrm{a}}, \dot{\mathbf{u}}_{\mathrm{a}}^{i}\right)\right|+\kappa\left\|\ddot{\mathbf{u}}^{i}-\ddot{\mathbf{u}}_{\mathrm{a}}^{i}\right\|, \\
\leq & \left|\frac{\partial}{\partial s} f_{\mathrm{n}}^{i}\left(s, \mathbf{U}, \dot{\mathbf{u}}^{i}\right)-\frac{\partial}{\partial s} f_{\mathrm{n}}^{i}\left(s, \mathbf{U}_{\mathrm{a}}, \dot{\mathbf{u}}_{\mathrm{a}}^{i}\right)+\dot{\mathbf{U}}^{+} \cdot \frac{\partial}{\partial \mathbf{U}} f_{\mathrm{n}}^{i}\left(s, \mathbf{U}, \dot{\mathbf{u}}^{i}\right)-\dot{\mathbf{U}}_{\mathrm{a}} \cdot \frac{\partial}{\partial \mathbf{U}_{\mathrm{a}}} f_{\mathrm{n}}^{i}\left(s, \mathbf{U}_{\mathrm{a}}, \dot{\mathbf{u}}_{\mathrm{a}}^{i}\right)\right|+\kappa\left\|\mathbf{r}^{i}\right\| \\
& \quad+\kappa\left\|\mathbf{r}_{\mathrm{a}}^{i}\right\|+\kappa\left\|\mathbf{f}^{i}\left(s, \mathbf{U}, \dot{\mathbf{u}}^{i}\right)-\mathbf{f}^{i}\left(s, \mathbf{U}_{\mathrm{a}}, \dot{\mathbf{u}}_{\mathrm{a}}^{i}\right)\right\| .
\end{aligned}
$$

This first term in the right handside is the modulus of some analytic function of $s, \mathbf{U}$, $\mathbf{U}$ which can be written in the form:

$$
\left(\mathbf{U}-\mathbf{U}_{\mathrm{a}}\right) \cdot G_{1}\left(s, \mathbf{U}-\mathbf{U}_{\mathrm{a}}, \dot{\mathbf{U}}-\dot{\mathbf{U}}_{\mathrm{a}}\right)+\left(\dot{\mathbf{U}}-\dot{\mathbf{U}}_{\mathrm{a}}\right) \cdot G_{2}\left(s, \mathbf{U}-\mathbf{U}_{\mathrm{a}}, \dot{\mathbf{U}}-\dot{\mathbf{U}}_{\mathrm{a}}\right) .
$$


But based on step 1, there exists a constant $C_{1}$ independent of $\mathbf{U}$ such that:

$$
\forall t \in[0, \eta[,
$$

$$
\begin{aligned}
& \mathbf{U}(t)-\mathbf{U}_{\mathrm{a}}(t)=C_{1} \int_{0}^{t}\left\|\mathbf{R}_{\mathrm{a}, \mathrm{n}}\right\|, \\
& \dot{\mathbf{U}}(t)-\dot{\mathbf{U}}_{\mathrm{a}}(t)=C_{1} \int_{0}^{t}\left\|\mathbf{R}_{\mathrm{a}, \mathrm{n}}\right\|,
\end{aligned}
$$

and some constants $C_{2}$ and $C_{3}$ independent of $\mathbf{U}$ such that:

$$
\begin{aligned}
\left|\frac{\partial}{\partial s} f_{\mathrm{n}}^{i}\left(s, \mathbf{U}, \dot{\mathbf{u}}^{i}\right)-\frac{\partial}{\partial s} f_{\mathrm{n}}^{i}\left(s, \mathbf{U}_{\mathrm{a}}, \dot{\mathbf{u}}_{\mathrm{a}}^{i}\right)+\mathbf{U}^{+} \cdot \frac{\partial}{\partial \mathbf{U}} f_{\mathrm{n}}^{i}\left(s, \mathbf{U}, \dot{\mathbf{u}}^{i}\right)-\mathbf{U}_{\mathrm{a}} \cdot \frac{\partial}{\partial \mathbf{U}_{\mathrm{a}}} f_{\mathrm{n}}^{i}\left(s, \mathbf{U}_{\mathrm{a}}, \dot{\mathbf{u}}_{\mathrm{a}}^{i}\right)\right| \\
+\kappa\left\|\mathbf{f}^{i}\left(s, \mathbf{U}, \dot{\mathbf{u}}^{i}\right)-\mathbf{f}^{i}\left(s, \mathbf{U}_{\mathrm{a}}, \dot{\mathbf{u}}_{\mathrm{a}}^{i}\right)\right\| \leq C_{2} \int_{0}^{s}\left\|\mathbf{R}_{\mathrm{a}, \mathrm{n}}\right\| \leq C_{3} \int_{0}^{s}\left|r_{\mathrm{a}, \mathrm{n}}^{i}\right|,
\end{aligned}
$$

where the fact $i \in D_{1}$ was used to obtain the second inequality. Recalling that $u_{\mathrm{n}}^{i} \mathbf{r}^{i} \equiv \mathbf{0}$, we have proved that there exists a constant $C_{5}$ independent of $\mathbf{U}$ such that:

$$
\left|r_{\mathrm{a}, \mathrm{n}}^{i}(t)\right|\left|u_{\mathrm{n}}^{i}(t)\right|-C_{5}\left|u_{\mathrm{n}}^{i}(t)\right| \int_{0}^{t}\left|r_{\mathrm{a}, \mathrm{n}}^{i}\right| \leq \int_{0}^{t} \dot{r}_{\mathrm{a}, \mathrm{n}}^{i} u_{\mathrm{n}}^{i}+\kappa\left(1+\mu_{i}\right) \int_{0}^{t}\left|r_{\mathrm{a}, \mathrm{n}}^{i}\right|\left|u_{\mathrm{n}}^{i}\right|+C_{5} \int_{0}^{t}\left|u_{\mathrm{n}}^{i}\right| \int_{0}^{s}\left|r_{\mathrm{a}, \mathrm{n}}^{i}\right| .
$$

Taking a smaller $\eta$ if necessary gives:

$$
\forall t \in] 0, \eta\left[, \quad\left|r_{\mathrm{a}, \mathrm{n}}^{i}(t)\right|-C_{5} \int_{0}^{t}\left|r_{\mathrm{a}, \mathrm{n}}^{i}\right|>0 .\right.
$$

The analyticity of $r_{\mathrm{a}, \mathrm{n}}^{i}(t)$ can be used as in [2] to estimate the derivative in terms of the function itself:

$$
\forall t \in] 0, \eta\left[, \quad\left|\dot{r}_{\mathrm{a}, \mathrm{n}}^{i}(t)\right| \leq \frac{m_{1}+\alpha_{i} t}{t}\left|r_{\mathrm{a}, \mathrm{n}}^{i}(t)\right|,\right.
$$

for some constant $\alpha_{i}$. We then obtain:

$$
\begin{aligned}
\left|\dot{r}_{\mathrm{a}, \mathrm{n}}^{i}(t)\right|+\kappa\left(1+\mu_{i}\right)\left|r_{\mathrm{a}, \mathrm{n}}^{i}(t)\right|+C_{5} \int_{0}^{t}\left|r_{\mathrm{a}, \mathrm{n}}^{i}\right| & \leq \frac{m_{1}+\alpha_{i} t}{t}\left|r_{\mathrm{a}, \mathrm{n}}^{i}(t)\right|+\kappa\left(1+\mu_{i}\right)\left|r_{\mathrm{a}, \mathrm{n}}^{i}(t)\right|+C_{5} \int_{0}^{t}\left|r_{\mathrm{a}, \mathrm{n}}^{i}\right|, \\
& \leq \frac{m_{1}+\beta_{i} t}{t}\left(\left|r_{\mathrm{a}, \mathrm{n}}^{i}(t)\right|-C_{5} \int_{0}^{t}\left|r_{\mathrm{a}, \mathrm{n}}^{i}\right|\right),
\end{aligned}
$$

for a larger constant $\beta_{i}$. Upon introducing the function $\psi_{i}(t)$ defined by:

$$
\psi_{i}(t) \stackrel{\text { def }}{=}\left(\left|r_{\mathrm{a}, \mathrm{n}}^{i}(t)\right|-C_{5} \int_{0}^{t}\left|r_{\mathrm{a}, \mathrm{n}}^{i}\right|\right) \frac{\left|u_{\mathrm{n}}^{i}(t)\right|}{t},
$$

which is non-negative and continuous on $\left[0, \eta\left[\right.\right.$ (it is continuous at $t=0$ because $u_{\mathrm{n}}^{i}(0)=0$ and $u_{\mathrm{n}}^{i}$ has a finite right-derivative at $t=0)$, we have proved:

$$
\forall t \in\left[0, \eta\left[, \quad t \psi_{i}(t) \leq\left(m_{1}+\beta_{i} t\right) \int_{0}^{t} \psi_{i}\right.\right.
$$

which entails:

$$
\forall t \in\left[0, \eta\left[, \quad \frac{\mathrm{d}}{\mathrm{d} t}\left(\frac{\mathrm{e}^{-\beta t}}{t^{m_{1}}} \int_{0}^{t} \psi_{i}\right) \leq 0\right.\right.
$$


so that $\int_{0}^{t} \psi_{i}$ is a non-positive function on $\left[0, \eta\left[\right.\right.$. By inequality (6), the same holds true of the function $\psi_{i}(t)$. Since this function is also non-negative, it vanishes identically and the same is therefore true of $u_{\mathrm{n}}^{i}(t)$. We have therefore proved that:

$$
\forall i \in D_{1}, \quad u_{\mathrm{n}}^{i} \equiv 0 \equiv u_{\mathrm{a}, \mathrm{n}}^{i}(t) .
$$

Step 3. The result proved in step 2 is used to improve the estimate in step 1.

Define $\widetilde{\mathbf{R}}_{\mathrm{a}}:\left[0, \eta\left[\mapsto \mathbb{R}^{n d}\right.\right.$ by:

$$
\widetilde{\mathbf{R}}_{\mathrm{a}}^{i}=\mid \begin{array}{rr}
0, & \text { if } i \in D_{1}, \\
\mathbf{R}_{\mathrm{a}}^{i}, & \text { if } i \notin D_{1} .
\end{array}
$$

Using the fact that $u_{\mathrm{n}}^{i} \equiv 0 \equiv u_{\mathrm{a}, \mathrm{n}}^{i}$, in the case of $i \in D_{1}$, which was proved in step 2, it is now proposed to prove that we can replace $\mathbf{R}_{\mathrm{a}}$ by $\widetilde{\mathbf{R}}_{\mathrm{a}}$ in the estimate proved in step 1 . Upon replaying the proof of step 1 , it can be readily checked that estimate (3) is now valid when $\mathbf{R}_{\mathrm{a}}$ is replaced by $\widetilde{\mathbf{R}}_{\mathrm{a}}$. Continuing to play the proof of step 1, we find estimate (4) unchanged. But we can then improve the estimate of the integral containing the normal reaction force as follows:

$$
\int_{[0, t]}\left\|\mathbf{R}_{\mathrm{n}}-\mathbf{R}_{\mathrm{a}, \mathrm{n}}\right\| \leq \sum_{i \in D_{1}} \int_{0}^{t}\left|r_{\mathrm{n}}^{i}-r_{\mathrm{a}, \mathrm{n}}^{i}\right|+\int_{0}^{t}\left\|\widetilde{\mathbf{R}}_{\mathrm{a}, \mathrm{n}}\right\|-\sum_{i \notin D_{1}} \int_{[0, t]} r_{\mathrm{n}}^{i} .
$$

With $i \in D_{1}$, the normal component of the equation of motion reads:

$$
0=r_{\mathrm{n}}^{i}-r_{\mathrm{a}, \mathrm{n}}^{i}+f_{\mathrm{n}}^{i}(t, \mathbf{U}, \dot{\mathbf{U}})-f_{\mathrm{n}}^{i}\left(t, \mathbf{U}_{\mathrm{a}}, \dot{\mathbf{U}}_{\mathrm{a}}\right),
$$

which gives:

$$
\sum_{i \in D_{1}} \int_{0}^{t}\left|r_{\mathrm{n}}^{i}-r_{\mathrm{a}, \mathrm{n}}^{i}\right| \leq \sqrt{n} \kappa\left(\left\|\mathbf{U}-\mathbf{U}_{\mathrm{a}}\right\|+\left\|\dot{\mathbf{U}}-\dot{\mathbf{U}}_{\mathrm{a}}\right\|\right) .
$$

Estimate (5) can therefore be improved as follows:

$$
\left\|\dot{\mathbf{U}}_{\mathrm{t}}^{+}(t)-\dot{\mathbf{U}}_{\mathrm{a}, \mathrm{t}}(t)\right\| \leq n(2 \sqrt{n}+1) \bar{\mu} \int_{0}^{t}\left\|\widetilde{\mathbf{R}}_{\mathrm{a}, \mathrm{n}}\right\|+(3 n \sqrt{n} \bar{\mu}+1) \kappa \int_{0}^{t}\left(\left\|\mathbf{U}-\mathbf{U}_{\mathrm{a}}\right\|+\left\|\dot{\mathbf{U}}^{+}-\dot{\mathbf{U}}_{\mathrm{a}}\right\|\right) .
$$

The remaining part of the proof of step 1 is replayed as previously and it can be readily checked that the conclusion of step 1 now holds true after replacing $\mathbf{R}_{\mathrm{a}}$ by $\widetilde{\mathbf{R}}_{\mathrm{a}}$.

\section{Step 4. Conclusion.}

If $\widetilde{\mathbf{R}}_{\mathrm{a}, \mathrm{n}}$ vanishes identically, then $\mathbf{U} \equiv \mathbf{U}_{\mathrm{a}}$ based on step 3. Let us therefore focus on the case where it does not vanish identically. Let $m_{2}>m_{1} \in \mathbb{N}$ be the order of the first non-zero term of its power series expansion at $t=0$. Let $D_{2}$ be the set of indexes of the corresponding particles:

$$
D_{2}=\left\{i \in\{1, \cdots, n\} \mid \exists \alpha_{i} \in \mathbb{R}, \quad r_{\mathrm{a}, \mathrm{n}}^{i}(t) \sim \alpha_{i} t^{m_{2}}\right\} .
$$

Replaying the proof of step 3 taking the result proved in step 3 into account, it can be readily proved that:

$$
\forall i \in D_{2}, \quad u_{\mathrm{n}}^{i} \equiv 0 \equiv u_{\mathrm{a}, \mathrm{n}}^{i} .
$$

Iterating this reasoning (a finite number of times) shows that $u_{\mathrm{n}}^{i} \equiv 0$ for all the indexes $i$ such that $u_{\mathrm{a}, \mathrm{n}}^{i} \equiv 0$. Replaying the proof of step 1 for the last time results in the same conclusion as step 1 with $\mathbf{R}_{\mathrm{a}}$ replaced by 0 . This means that $\mathbf{U}$ equals identically $\mathbf{U}_{\mathrm{a}}$ on $[0, \eta[$. 
Corollary 4.3. Theorem 2.1 holds true.

Proof. Using the local existence of a solution to problem $\mathcal{P}_{\mathrm{u}}$ given by Proposition 4.1, and the local uniqueness in $M M A$, given by Theorem 4.2, we obtain a maximal solution $\mathbf{U}$ which is defined either in a sub-interval $\left[0, t_{\max }\left[\right.\right.$, for some $\left.\left.t_{\max } \in\right] 0, T\right]$, or in $[0, T]$. We now have to prove that it is defined on $[0, T]$. To reach this conclusion, it suffices to prove that, if the maximal solution was defined only in a sub-interval $\left[0, t_{\max }[\right.$, then, the total variation of the right-velocity $\dot{\mathbf{U}}^{+}$over $\left[0, t_{\max }\right.$ [ will be finite, because in this case, it would be possible to extend $\mathbf{U}$ beyond $\left[0, t_{\max }\right.$ [, resulting in a contradiction. Let us therefore assume that the maximal solution is defined only in $\left[0, t_{\max }[\right.$.

First, we have noted above that $\mathbf{R}_{\mathrm{n}} \cdot\left(\dot{\mathbf{U}}_{\mathrm{n}}^{+}+\dot{\mathbf{U}}_{\mathrm{n}}^{-}\right)$is a non-positive measure, in view of the contact condition, the equation of motion and the impact law. Also, based on the Coulomb friction law, $\mathbf{R}_{\mathrm{t}} \cdot \dot{\mathbf{U}}_{\mathrm{t}}^{+}$is a non-positive measure. Let $\mathcal{T}$ be the countable subset consisting of the instants $t \in\left[0, t_{\max }\right.$ [ at which the tangential velocity is discontinuous: $\dot{\mathbf{U}}_{\mathrm{t}}^{+}(t) \neq \dot{\mathbf{U}}_{\mathrm{t}}^{-}(t)$. On $\left[0, t_{\max }\left[\backslash \mathcal{T}\right.\right.$, the measure $\mathbf{R}_{\mathrm{t}} \cdot\left(\dot{\mathbf{U}}_{\mathrm{t}}^{+}+\dot{\mathbf{U}}_{\mathrm{t}}^{-}\right)$equals $\mathbf{R}_{\mathrm{t}} \cdot \dot{\mathbf{U}}_{\mathrm{t}}^{+}$which is nonpositive. At each instant $t \in \mathcal{T}$, using the equation of motion along with the Coulomb friction law, it can be readily checked that the measure $\mathbf{R}_{\mathrm{t}} \cdot\left(\dot{\mathbf{U}}_{\mathrm{t}}^{+}+\dot{\mathbf{U}}_{\mathrm{t}}^{-}\right)$has a negative atom. Finally, $\mathbf{R} \cdot\left(\dot{\mathbf{U}}^{+}+\dot{\mathbf{U}}^{-}\right)$is a non-negative measure. So, multiplying the equation of motion by $\left(\dot{\mathbf{U}}^{+}+\dot{\mathbf{U}}^{-}\right) / 2$ and integrating over $\left.] 0, t\right](t \in] 0, t_{\max }[)$ gives the energy inequality:

$$
\begin{aligned}
\frac{1}{2}\left\|\dot{\mathbf{U}}^{+}(t)\right\|^{2} & \leq \frac{1}{2}\left\|\mathbf{V}_{0}\right\|^{2}+\int_{0}^{t} \dot{\mathbf{U}}^{+} \cdot \mathbf{F}\left(s, \mathbf{U}, \dot{\mathbf{U}}^{+}\right), \\
& \leq \frac{1}{2}\left\|\mathbf{V}_{0}\right\|^{2}+\int_{0}^{t}\left\|\mathbf{F}\left(s, \mathbf{U}_{0}, \mathbf{0}\right)\right\|+\kappa \int_{0}^{t}\left\|\dot{\mathbf{U}}^{+}\right\|\left(\left\|\mathbf{U}-\mathbf{U}_{0}\right\|+\left\|\dot{\mathbf{U}}^{+}\right\|\right), \\
& \leq \frac{1}{2}\left\|\mathbf{V}_{0}\right\|^{2}+\int_{0}^{t}\left\|\mathbf{F}\left(s, \mathbf{U}_{0}, \mathbf{0}\right)\right\|+\kappa(1+T) \int_{0}^{t}\left\|\dot{\mathbf{U}}^{+}\right\|^{2} .
\end{aligned}
$$

Based on Gronwall's lemma, this energy inequality means that the right-velocity $\dot{\mathbf{U}}^{+}$is bounded over the interval $\left[0, t_{\max }\left[\right.\right.$. Next, integrating the first component of the equation of motion over $[0, t](t \in] 0, t_{\max }[)$, we obtain:

$$
\int_{[0, t]} \mathbf{R}_{\mathrm{n}}=\dot{\mathbf{U}}_{\mathrm{n}}^{+}(t)-\mathbf{V}_{0 \mathrm{n}}+\int_{0}^{t} \mathbf{f}_{\mathrm{n}}\left(s, \mathbf{U}, \dot{\mathbf{U}}^{+}\right)
$$

which shows, since the right-velocity $\dot{\mathbf{U}}^{+}$is bounded over $\left[0, t_{\max }\left[\right.\right.$ and since each component of $\mathbf{R}_{\mathrm{n}}$ is a nonpositive measure, that the measure $\mathbf{R}_{\mathrm{n}}$ is bounded:

$$
\int_{\left[0, t_{\max }[\right.}\left\|\mathbf{R}_{\mathrm{n}}\right\|<\infty
$$

But Coulomb friction law implies that:

$$
\forall i, \quad\left\|\mathbf{r}_{\mathrm{t}}^{i}\right\| \leq \mu_{i}\left|r_{\mathrm{n}}^{i}\right|
$$

and therefore:

$$
\int_{\left[0, t_{\max }[\right.}\left\|\mathbf{R}_{\mathrm{t}}\right\|<\infty .
$$

Going back to the equation of motion, we obtain:

$$
\int_{\left[0, t_{\max }[\right.}\|\ddot{\mathbf{U}}\|<\infty
$$

which is the expected conclusion. 


\section{HANDLING CURVED OBSTACLES}

In this section, we investigate the situation where each obstacle is defined by an analytic hypersurface $S_{i}$ instead of a hyperplane. More specifically, it is required that for each $\mathbf{x}$ on the hypersurface $S_{i}$, there should exist a neighborhood $\mathcal{N}$ of $\mathbf{x}$ in $\mathbb{R}^{d}$, an open set $\mathcal{O} \subset \mathbb{R}^{d}$ and an analytic diffeomorphism $\psi: \mathcal{O} \rightarrow \mathcal{N}$ such that $\psi^{-1}\left(S_{i} \cap \mathcal{N}\right) \subset\{0\} \times \mathbb{R}^{d-1}$ and points $\mathbf{x}^{\prime} \in \mathcal{N}$ lying outside the obstacle are characterized by the condition $\left.\psi^{-1}\left(\mathbf{x}^{\prime}\right) \in\right]-\infty, 0\left[\times \mathbb{R}^{d-1}\right.$. In other words, at each $\mathbf{x} \in S_{i}$, there must exist an analytic local curvilinear coordinate system in $\mathbb{R}^{d}$ such that the non-penetration condition is the requirement for the first coordinate to be non-positive. Taking $\mathbf{e}_{n}$ to denote the first vector in the natural basis, it is always possible to construct an analytic local curvilinear coordinate system such that the vector field $\mathbf{e}_{\mathrm{n}}$ on $S_{i}$ is everywhere orthogonal to $S_{i}$. This choice will be systematically made, so that the first coordinate of this curvilinear coordinate system is the coordinate 'normal' to the obstacle.

In particular, it is possible to find a local curvilinear coordinate system of this kind for each particle in a neighborhood of a given arbitrary initial condition imposed on the system. Upon using the natural basis of that coordinate system systematically to express velocities, accelerations and forces, it turns out that the new formulation of problem $\mathcal{P}_{\mathbf{u}}$ in the case of curved obstacles reads as follows.

Problem $\mathcal{P}_{\mathbf{u}}$. Find $\left.\left.T_{0} \in\right] 0, T\right], \mathbf{U} \in \operatorname{MMA}\left(\left[0, T_{0}\right] ; \mathbb{R}^{n d}\right)$ and $\mathbf{R} \in \mathcal{M}\left(\left[0, T_{0}\right] ; \mathbb{R}^{n d}\right)$ such that:

- $\mathbf{U}(0)=\mathbf{U}_{0} \quad ; \quad \dot{\mathbf{U}}^{+}(0)=\mathbf{V}_{0}$

- $\forall i \in\{1,2, \ldots, n\}, \quad \forall k \in\{1,2, \ldots, d\}$,

$$
\ddot{u}_{k}^{i}=f_{k}^{i}(t, \mathbf{U}, \dot{\mathbf{U}})-\sum_{p, q=1}^{d}{ }^{i} \Gamma_{p q}^{k} \dot{u}_{p}^{i} \dot{u}_{q}^{i}+r_{k}^{i}, \quad \text { in }\left[0, T_{0}\right]
$$

- $\forall i \in\{1,2, \ldots, n\}, \quad \forall t \in] 0, T_{0}[$,

$$
u_{\mathrm{n}}^{i} \leq 0, \quad r_{\mathrm{n}}^{i} \leq 0, \quad u_{\mathrm{n}}^{i} r_{\mathrm{n}}^{i}=0
$$

- $\forall i \in\{1,2, \ldots, n\}, \quad \forall \mathbf{v} \in C^{0}\left(\left[0, T_{0}\right] ; \mathbb{R}^{d-1}\right)$,

$$
\int_{\left[0, T_{0}\right]}\left[\mathbf{r}_{\mathrm{t}}^{i} \cdot\left(\mathbf{v}-\dot{\mathbf{u}}_{\mathrm{t}}^{i+}\right)-\mu_{i} r_{\mathrm{n}}^{i}\left(\|\mathbf{v}\|-\left\|\dot{\mathbf{u}}_{\mathrm{t}}^{i+}\right\|\right)\right] \geq 0,
$$

- $\forall i \in\{1,2, \ldots, n\}, \quad \forall t \in] 0, T_{0}[$,

$$
u_{\mathrm{n}}^{i}(t)=0 \quad \Longrightarrow \quad \dot{u}_{\mathrm{n}}^{i+}(t)=-e_{i} \dot{u}_{\mathrm{n}}^{i-}(t),
$$

(impact law),

where the ${ }^{i} \Gamma_{p q}^{k}(p, q, k \in\{1, \ldots, d\})$ denote the Christoffel symbols of the curvilinear coordinate system involving particle $i$.

This problem is formally identical to that which arose in the case of straight obstacles, since the Christoffel symbols depend analytically on the configuration, except that one must construct $T_{0}$ so that the solution does not escape from $\mathcal{O}$. This is done as follows. First, since $\mathcal{O}$ is open, one can find $\rho_{0}>0$ such that the closed ball $\bar{B}\left(\mathbf{U}_{0}, \rho_{0}\right)$ of $\mathbb{R}^{\text {nd }}$ is contained in $\mathcal{O}$. Next, consider any solution $(\mathbf{U}, \mathbf{R})$ of problem $\mathcal{P}_{\mathbf{u}}$. Multiplying the 
equation of motion by $\left(U^{+}+U^{-}\right) / 2$ and integrating gives:

$$
\begin{aligned}
\frac{1}{2}\left\|\dot{\mathbf{U}}^{+}(t)\right\|^{2} & \leq \frac{1}{2}\left\|\mathbf{V}_{0}\right\|+\int_{0}^{t} \mathbf{F}\left(s, \mathbf{U}, \dot{\mathbf{U}}^{+}\right) \cdot \dot{\mathbf{U}}^{+} \mathrm{d} s+\int_{[0, t]} \mathbf{R} \cdot\left(\dot{\mathbf{U}}^{+}+\dot{\mathbf{U}}^{-}\right) / 2, \\
& \leq \frac{1}{2}\left\|\mathbf{V}_{0}\right\|^{2}+\int_{0}^{t} \dot{\mathbf{U}}^{+} \cdot \mathbf{F}\left(s, \mathbf{U}, \dot{\mathbf{U}}^{+}\right), \\
& \leq \frac{1}{2}\left\|\mathbf{V}_{0}\right\|^{2}+\int_{0}^{t}\left\|\mathbf{F}\left(s, \mathbf{U}_{0}, \mathbf{0}\right)\right\|+\kappa \int_{0}^{t}\left\|\dot{\mathbf{U}}^{+}\right\|\left(\left\|\mathbf{U}-\mathbf{U}_{0}\right\|+\left\|\dot{\mathbf{U}}^{+}\right\|\right), \\
& \leq \frac{1}{2}\left\|\mathbf{V}_{0}\right\|^{2}+\int_{0}^{t}\left\|\mathbf{F}\left(s, \mathbf{U}_{0}, \mathbf{0}\right)\right\|+\kappa(1+T) \int_{0}^{t}\left\|\dot{\mathbf{U}}^{+}\right\|^{2} .
\end{aligned}
$$

Using Gronwall's lemma yields the following estimate for the velocity:

$$
\frac{1}{2}\left\|\dot{\mathbf{U}}^{+}(t)\right\|^{2} \leq \mathrm{e}^{\kappa T(1+T)}\left(\frac{1}{2}\left\|\mathbf{V}_{0}\right\|^{2}+\int_{0}^{T}\left\|\mathbf{F}\left(s, \mathbf{U}_{0}, \mathbf{0}\right)\right\|\right) .
$$

Setting:

$$
\mathbf{V}_{\max } \stackrel{\text { def }}{=} \mathrm{e}^{\kappa T(1+T) / 2}\left(\left\|\mathbf{V}_{0}\right\|^{2}+2 \int_{0}^{T}\left\|\mathbf{F}\left(s, \mathbf{U}_{0}, \mathbf{0}\right)\right\|\right)^{1 / 2},
$$

we can see that it suffices to choose:

$$
T_{0} \stackrel{\text { def }}{=} \frac{\rho_{0}}{\mathbf{V}_{\max }},
$$

to ensure that:

$$
\forall t \in\left[0, T_{0}\right], \quad\left\|\mathbf{U}(t)-\mathbf{U}_{0}\right\| \leq \rho_{0},
$$

and therefore that $\mathbf{U}(t) \in \mathcal{O}$. It is then quite easy to replay the proof of Theorem 2.1 to obtain a unique solution $\mathbf{U} \in \operatorname{MMA}\left(\left[0, T_{0}\right] ; \mathbb{R}^{n d}\right)$ for problem $\mathcal{P}_{\mathbf{u}}$ with curved obstacles. Lastly a replay of the proof of corollary 4.3 gives the required extension of Theorem 2.1 to the case of curved (analytic) obstacles.

\section{Appendix A. Bounded variation, Composite mappings And DERIVAtives}

Let $v:[a, b] \mapsto \mathbb{R}$ be a function with bounded variation. The function $v$ is necessarily bounded and takes values in, say, $[m, M]$. Let $f:[m, M] \mapsto \mathbb{R}$ be a function of class $C^{1}$, which is therefore Lispchitz-continuous of modulus, say, $\kappa$. It is obvious that the composite mapping $f \circ v:[a, b] \mapsto \mathbb{R}$ has bounded variation. Denoting its Stieljes measure (see [11], the Stieljes measure of a function with bounded variation is nothing but the distributional derivative which turns out in this case to be a measure) by $\mathrm{d}(f \circ v)$, it would be natural to expect a chain rule like:

$$
\mathrm{d}(f \circ v)=\left(f^{\prime} \circ v\right) \mathrm{d} v
$$

to hold true. But it fails to be true, in general.

Counter-example. Take $v$ as the restriction of the Heaviside function to $[-1,1]$ (that is, taking the value 0 on $[-1,0[$ and 1 on $[0,1])$. Set:

$$
f(x)=\frac{1-\cos (\pi x)}{2}
$$

Denoting by $\delta$ the Dirac measure at 0 , it is readily checked that:

$$
\begin{gathered}
\mathrm{d}(f \circ v)=\delta, \\
f^{\prime}(v(0-)) \mathrm{d} v=f^{\prime}(v(0+)) \mathrm{d} u=f^{\prime}(v(0)) \mathrm{d} v=0 .
\end{gathered}
$$

However, an inequality can still be proved very easily as seen in Proposition A.1. A direct proof of that proposition is provided, although it is also a corollary of a general chain rule formula established in [1]. 
Proposition A.1. Let $v$ and $f$ be as stated above. Then, the following inequality between modulus measures:

$$
|\mathrm{d}(f \circ v)| \leq \kappa|\mathrm{d} v|
$$

holds true.

Proof. Consider an arbitrary $] c, d[\subset[a, b]$. We have:

$$
\left|\int_{] c, d[} \mathrm{d}(f \circ v)\right|=|f(v(d-))-f(v(c+))| \leq \kappa|v(d-)-v(c+)|=\kappa\left|\int_{] c, d[} \mathrm{d} v\right| .
$$

Since $c, d$ are arbitrary, it entails the expected inequality.

Actually, the result which is needed in the paper (proof of Thm. 4.2) is a slight extension of this last proposition whose proof is left to the reader.

Proposition A.2. Let $v:[a, b] \mapsto[m, M]$ be a function with bounded variation, $u:[a, b] \mapsto[m, M]$ be an absolutely continuous function (that is, a function with bounded variation whose distributional derivative is an integrable function), and $f:[m, M]^{2} \mapsto \mathbb{R}$ be a function of class $C^{1}$ whose Lipschitz modulus is denoted by $\kappa$. Set $g(x)=f(u(x), v(x))$. Then, $g$ has bounded variation and:

$$
|\mathrm{d} g| \leq\left|\frac{\partial}{\partial u} f(u(x), v(x)) \mathrm{d} u\right|+\kappa|\mathrm{d} v| .
$$

\section{REFERENCES}

[1] L. Ambrosio and G. Dal Maso, A general chain rule for distributional derivatives. Proc. Amer. Math. Soc. 108 (1990) $691-702$.

[2] P. Ballard, The dynamics of discrete mechanical systems with perfect unilateral constraints. Arch. Ration. Mech. Anal. 154 (2000) 199-274.

[3] P. Ballard and S. Basseville, Existence and uniqueness for dynamical unilateral contact with coulomb friction: a model problem, ESAIM: M2AN 39 (2005) 59-77.

[4] H. Brezis, Opérateurs Maximaux Monotones et Semi-groupes de Contractions dans les Espaces de Hilbert. North-Holland Publishing Company (1973).

[5] C. Eck, J. Jarušek and M. Krbec, Unilateral Contact Problems in Mechanics. Variational Methods and Existence Theorems. Monographs \& Textbooks in Pure \& Appl. Math. No. 270 (ISBN 1-57444-629-0). Chapman \& Hall/CRC, Boca Raton (2005).

[6] A. Klarbring, Ingenieur-Archiv 60 (1990) 529-541.

[7] M.D.P. Monteiro Marques, Differential Inclusions in Nonsmooth Mechanical Problems. Birkhaüser, Basel-Boston-Berlin (1993).

[8] J.J. Moreau, Standard inelastic shocks and the dynamics of unilateral constraints, in Unilateral problems in structural analysis, edited by G. Del Piero and F. Maceri. Springer-Verlag, Wien-New-York (1983) 173-221.

[9] J.J. Moreau, Dynamique de systèmes à liaisons unilatérales avec frottement sec éventuel: essais numériques, Note Technique No 85-1, LMGC, Montpellier (1985).

[10] J.J. Moreau, Unilateral contact and dry friction in finite freedom dynamics, in Nonsmooth Mechanics and Applications, CISM Courses and Lectures No 302, edited by J.J. Moreau and P.D. Panagiotopoulos. Springer-Verlag, Wien-New-York (1988) 1-82.

[11] J.J. Moreau, Bounded variation in time, in Topics in Non-smooth Mechanics, edited by J.J. Moreau, P.D. Panagiotopoulos and G. Strang. Birkhaüser Verlag, Basel-Boston-Berlin (1988) 1-74.

[12] P. Painlevé, Sur les lois du frottement de glissement. C.R. Acad. Sci. (Paris) 121 (1895) 112-115.

[13] D. Percivale, Uniqueness in the Elastic Bounce Problem, I, J. Diff. Eqs. 56 (1985) 206-215.

[14] M. Schatzman, A Class of Nonlinear Differential Equations of Second Order in Time, Nonlinear Analysis. Theory, Methods Appl. 2 (1978) 355-373.

[15] M. Schatzman, Uniqueness and continuous dependence on data for one dimensional impact problems. Math. Comput. Modell. 28 (1998) 1-18. 